\title{
Comparative Statics by Adaptive Dynamics and The Correspondence Principle *
}

\author{
Federico Echenique $^{\dagger}$
}

January 18,2000

\begin{abstract}
This paper formalizes the relation between comparative statics and the out-of-equilibrium explanation for how a system evolves after a change in parameters. The paper has two main results. First, an increase in an exogenous parameter sets off learning dynamics that involve larger values of the endogenous variables. Second, equilibrium selections that are not monotone increasing in the exogenous variables must be predicting unstable equilibria. Moreover, under some conditions monotone comparative statics and stability are equivalent.
\end{abstract}

JEL Classification: C61, C62, C72, C73

Keywords: Monotone comparative statics, supermodularity, strategic complements, learning, correspondence principle.

${ }^{*}$ Discussions with Ilya Segal and Chris Shannon were very important for this work, I am very grateful for all their help. For comments and advice, I also thank Robert Anderson, Juan Dubra, Néstor Gandelman, Ernesto López Córdova, Marcelo Moreira, Charles Pugh, Matthew Rabin, Tarun Sabarwal and Miguel Villas-Boas. Financial support from Prof. Rabin is very gratefully acknowledged.

${ }^{\dagger}$ Department of Economics, University of California at Berkeley, 549 Evans Hall \# 3880. Berkeley, CA 947203880, U.S.A. email: fede@econ. berkeley.edu and Facultad de Ciencias Sociales, Universidad de la República 


\section{Introduction}

We find ourselves confronted with this paradox: in order for the comparative statics analysis to yield fruitful results, we must first develop a theory of dynamics.

Samuelson (1947, p. 262)

The intuition behind comparative statics results is usually dynamic in nature. The economic explanation for why differences in endogenous variables result from variations in exogenous variables often takes the form of some sequential adjustment process. For example, consider Milgrom, Qian, and Roberts's (1991) account of technological and organizational changes in modern manufacturing: "... the falling costs of high-speed data communication, data processing, and flexible, multitask equipment lead to increases in the directly affected activities, which through a web of complementarities then lead to increases in a set of related activities as well."

Despite the prevalence of dynamic economic explanations, the formal analysis is static. The existing results on monotone comparative statics of equilibria can be summarized in the statement of Milgrom and Roberts's (1990, MR hereafter) theorem that the largest and smallest equilibria in a parameterized supermodular game are increasing in the parameter. ${ }^{1}$ In any game, however, we can have a large number of equilibria and there are a priori no reasons to expect the largest or smallest equilibrium to be played. One possibility is using out-ofequilibrium dynamics to select the equilibrium played after a change in parameters.

Consider the following game. There are two agents that choose simultaneously the effort level $x \in[0,1]$ that they put into a common task. They use a common technology whose productivity is indexed by a real number $t$, so that a higher value of $t$ implies a higher productivity. Let $\beta_{i}(x, t)$ be worker $i$ 's optimal choice of effort when the other agent chooses $x$, i.e. her best response function. Then, $\beta(x, t)=\beta_{1}\left(\beta_{2}(x, t), t\right)$ is called the aggregate best response function and the Nash equilibria of the game coincide with the fixed points of $\beta(., t)$. Assume that the players' efforts are complementary so that $\beta_{i}(x, t)$ is increasing in $x$ and that higher productivity makes each agent want to work harder so that $\beta_{i}(x, t)$ is increasing in $t$. Figure 1

\footnotetext{
${ }^{1}$ Lippman, Mamer, and McCardle (1987) were the first to present a result of this kind. They prove that the parameterized set of fixed points admits a monotone selection. Sobel (1988) developed the result for extremal equilibria independently of MR. Milgrom and Shannon (1994) generalize the result to games of ordinal strategic complementarities. See Topkis (1998) for an exposition of the theory.
} 

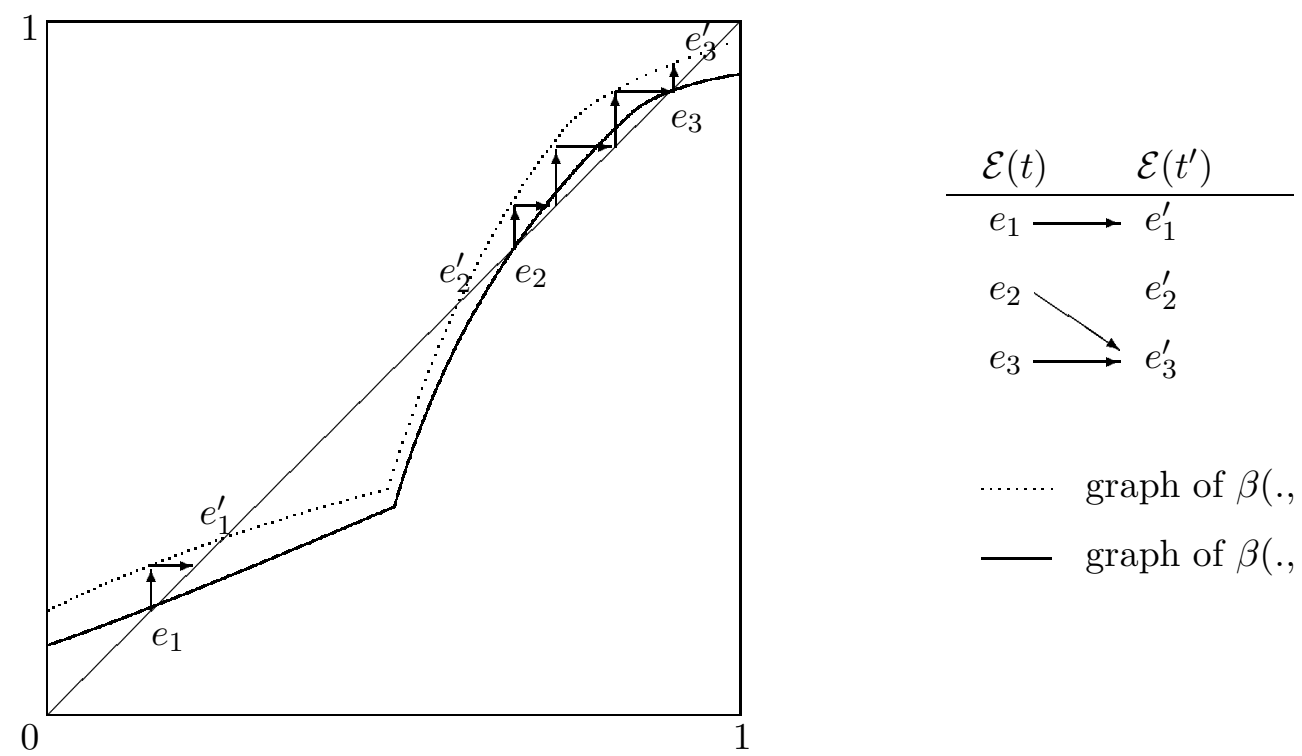

raph of $\beta\left(., t^{\prime}\right)$

- graph of $\beta(., t)$

Figure 1: An increase in $t$

shows a typical aggregate best response function for a game of strategic complementarities like the one described. The dotted graph represents the game after an increase in the parameter and $\mathcal{E}(t)$ denotes the set of equilibria for parameter $t$.

Figure 1 shows that, in accordance with the MR result, the smallest and largest equilibria increase after an increase in $t$. However, we might expect agents to be playing equilibrium $e_{2}$. In this case the results for extremal equilibria are silent. On the other hand, for small parameter changes the Implicit Function Theorem gives local comparative statics at each equilibria. If we expect $e_{2}$ to be played we obtain a conclusion opposite to MR's result: $e_{2}^{\prime}$, the closest "new" equilibrium, is smaller than $e_{2} \cdot{ }^{2}$

On the other hand, Samuelson's Correspondence Principle (CP) says that, selecting equilibria that are stable for some reasonable out-of-equilibrium dynamics gives unambiguous comparative statics results. Note that $e_{2}$ and $e_{2}^{\prime}$ are unstable for the "Cournot best response dynamics", $x_{n}=\beta\left(x_{n-1}, t\right){ }^{3}$

It seems then that, unless there is a reason for selecting extremal equilibria, the old meth-

\footnotetext{
${ }^{2}$ Milgrom and Roberts (1994, p. 455) mention this fact and say that a local application of their result predicts the decrease.

${ }^{3}$ In Samuelson's (1947) analysis, the comparative statics are obtained using the implicit function theorem on parameterized "equilibrium conditions" (a system of equations) and the correspondence principle imposes signs on the resulting derivatives by requiring the equilibrium to be stable with respect to some specified dynamics.
} 
ods of comparative statics coupled with the selection criterion of choosing stable equilibria have an advantage over the new literature. ${ }^{4}$ In the example, since all increasing selections of equilibria pick stable points, the Implicit Function Theorem coupled with the CP yields unambiguous comparative statics results. MR's result does not give a conclusive answer to how the endogenous variables change after an increase in $t$.

This paper shows that if the new methods are endowed with the CP then they too yield unambiguous comparative statics. Two main ideas are developed. These ideas can be illustrated using the above example:

1. If the workers are at an equilibrium and there is an increase in productivity, then each one will desire to increase her effort. If both agents realize this, then, because of complementarity between efforts, they will want to further increase their efforts. This argument suggests that any prediction of play after an increase in the parameter should involve larger efforts than the original equilibrium. For example, consider the "Cournot best response dynamics" where in each round, players select a best response to last round's play. In Figure 1 it is easy to see that this dynamic in the $t^{\prime}$-game starting at any of the three equilibria for the $t$-game converges to a larger equilibrium (this is indicated by the arrows in Figure 1).

In the paper I show that, for a general class of dynamics, play will be larger after an increase in the parameter in models with complementarity between the state variables. In addition, considering a class of adjustment dynamics similar to that used in Milgrom and Roberts (1990,1991) and Milgrom and Shannon (1994), convergence to larger equilibria can be ensured.

2. What is wrong with equilibrium $e_{2}$ ? Since $e_{2}$ has arbitrarily close smaller equilibria corresponding to larger parameter values, then, by starting at any of these smaller equilibria, decreasing the parameter to $t$ and reasoning as in Item 1 we obtain a prediction of play that is yet smaller. For this reason, $e_{2}$ must be unstable under any dynamics obtained by

\footnotetext{
${ }^{4}$ Pareto optimality or coalition proofness are reasons to select extremal equilibria in e.g. games of coordination failures. But also in many of these examples the interesting feature of the model is that the socially optimal equilibria may not be selected (like in network externalities or macroeconomic coordination failures).
} 
reasoning as in Item 1.

On the other hand, by looking at the change from $e_{1}$ to $e_{1}^{\prime}$ and from $e_{3}$ to $e_{3}^{\prime}$ it can be seen that these selections are increasing and select stable equilibria. Hence, in this picture, stability is the same as monotonicity. This turns out to be a general result, as I show in Section 4 .

Since instability of equilibria usually leads game theorists (and probably players too) to doubt that players will select a particular equilibrium, my results imply that we should be at least suspicious about a selection of equilibria that is not monotone in the parameter. The CP gives monotone comparative statics results as long as we agree to refine away unstable equilibria.

Thus, if endowed with the $\mathrm{CP}$, the recent methods for comparative statics of equilibria yield unambiguous conclusions. In this sense, the $\mathrm{CP}$ presented in this paper has the same advantage over Samuelson's as the new comparative statics methods represented by the theorems of Topkis and Milgrom and Shannon have over the old (see Milgrom and Shannon (1994) for a discussion). No convexity or smoothness of the maps or spaces involved is needed, no Inada conditions, no need to restrict to Euclidean spaces. Given the importance of increasing returns and other non-convexities in many areas of economics, it is important to have methods that do not require convexity. If the example presented above had discrete choice sets, the Implicit Function Theorem could not have been used. In Section 6.2 I present Farrell and Saloner's (1985) network externalities model as an example where none of the existing comparative statics methods are useful.

A final advantage of this version of the $\mathrm{CP}$ is that the dynamics used encompass a wide array of behavioral assumptions. In this sense the results are robust to the specification of out-of-equilibrium dynamics.

Samuelson gave examples of economic models where he was able to obtain local comparative statics conclusions by imposing stability. Some of Samuelson's applications are covered by my results on monotone maps. The others are explained in Section 5: in non monotone onedimensional problems like partial equilibrium and two good general equilibrium models the 
$\mathrm{CP}$ works. In general multidimensional cases the principle need not yield comparative statics conclusions. ${ }^{5}$ Here I extend the applicability of the CP to arbitrary lattices (without restrictions on dimensions) as long as complementarity is built into the model.

The paper proceeds as follows. Section 2 presents basic definitions and notation. Section 3 contains the results on convergence to larger equilibrium values. Section 4 presents two versions of the CP and some partial converse results. Section 5 discusses the scope of the CP. Section 6 contains an illustration of the results through three applications and Section 7 concludes. Except for a few that are both short and give insight, all proofs are in Section 8.

\section{Definitions}

\subsection{Standard Definitions}

A detailed discussion of the concepts defined in this subsection can be found in Topkis (1998). A set $X$ with a transitive, reflexive, antisymmetric binary relation $\preceq$ is a lattice if whenever $x, y \in X$, both $x \wedge y=\inf \{x, y\}$ and $x \vee y=\sup \{x, y\}$ exist in $X$. It is complete if for every nonempty subset $A$ of $X, \inf A, \sup A$ exist in $X$. A nonempty subset $A$ of $X$ is a sublattice if for all $x, y \in A, x \wedge_{X} y, x \vee_{X} y \in A$, where $x \wedge_{X} y$ and $x \vee_{X} y$ are obtained taking the infimum and supremum as elements of $X$ (as opposed to using the relative order on $A$ ). A nonempty

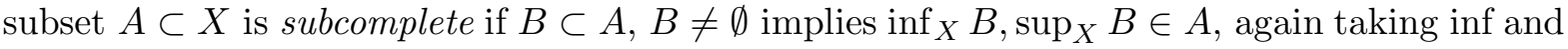
sup of $B$ as a subset of $X$. For two subsets $A, B$ of $X$, say that $A$ is smaller than $B$ in the strong set order, denoted $A \leq_{s} B$, if $a \in A, b \in B$ implies $a \wedge b \in A, a \vee b \in B$. The order interval topology on a lattice is obtained by taking the closed intervals $[x, y]=\{z \in X: x \preceq z \preceq y\}$ as a sub-basis for the closed sets. All lattices in the paper will be endowed with the order interval topology. For any subset $A$ of a topological space, $A^{o}$ will denote the set of interior points of $A$. I will use $\preceq$ to denote the order on lattices and $\leq$ to refer to the order on indexes and $\mathbf{R}$. If $X$ is a lattice, a function $f: X \rightarrow \mathbf{R}$ is quasisupermodular if for any $x, y \in X, f(x) \geq f(x \wedge y)$ implies $f(x \vee y) \geq f(y)$ and $f(x)>f(x \wedge y)$ implies $f(x \vee y)>f(y)$. Let $T$ be a partially ordered set. A function $f: X \times T \rightarrow \mathbf{R}$ satisfies the single crossing condition in $(x, t)$ if whenever $x \prec x^{\prime}$

\footnotetext{
${ }^{5}$ The negative result is not new, Arrow and Hahn (1971) argued that for general equilibrium analysis, the CP was not of much use; imposing stability alone does not guarantee unambiguous comparative statics predictions.
} 
and $t \prec t^{\prime}, f(x, t) \leq f\left(x^{\prime}, t\right)$ implies that $f\left(x, t^{\prime}\right) \leq f\left(x^{\prime}, t^{\prime}\right)$ and $f(x, t)<f\left(x^{\prime}, t\right)$ implies that $f\left(x, t^{\prime}\right)<f\left(x^{\prime}, t^{\prime}\right)$. Say that $f$ satisfies the strict single crossing condition in $(x, t)$ if whenever $x \prec x^{\prime}$ and $t \prec t^{\prime}, f(x, t) \leq f\left(x^{\prime}, t\right)$ implies that $f\left(x, t^{\prime}\right)<f\left(x^{\prime}, t^{\prime}\right)$.

\subsection{Definitions and Notation for this Paper}

Let $X$ be a lattice. Say that a correspondence $\phi: X \rightarrow X$ is weakly increasing if for any $x, x^{\prime} \in X$ with $x \prec x^{\prime}$ we have $\inf \phi(x) \preceq \inf \phi\left(x^{\prime}\right)$ and $\sup \phi(x) \preceq \sup \phi\left(x^{\prime}\right)$. Also, say that $\phi$ is strongly increasing if for any $x, x^{\prime} \in X$ with $x \prec x^{\prime}$ we have $\sup \phi(x) \preceq \inf \phi\left(x^{\prime}\right) .{ }^{6}$ Let $T$ be a partially ordered set. An increasing family of correspondences $\left(\phi_{t}: t \in T\right)$ is a correspondence $\phi: X \times T \rightarrow X$ such that $x \mapsto \phi_{t}(x)$ is weakly increasing and $t \mapsto \phi_{t}(x)$ is strongly increasing. If, in addition, $x \mapsto \phi_{t}(x)$ is upper hemicontinuous and subcomplete sublattice valued then it will be called an increasing family of uhc correspondences. If $\left(\phi_{t}: t \in T\right)$ is an increasing family of uhc correspondences and $\phi_{t}$ is single valued for all $t$ then I will call it an increasing family of uhc functions. Let $\mathcal{E}=\{x \in X: x \in \phi(x)\}$ be the set of fixed points of $\phi$. When $\left(\phi_{t}: t \in T\right)$ is a family of correspondences, the notation will be $\mathcal{E}(t)=\left\{x \in X: x \in \phi_{t}(x)\right\}$. Let $\phi(x)=\inf \phi(x)$ and $\bar{\phi}(x)=\sup \phi(x)$ for all $x$.

Given a sequence $\left\{x_{k}\right\}$ in $X$, let $H_{k}^{\gamma}=\left\{x_{k-\gamma}, \ldots x_{k-1}\right\}$ denote the history of length $\gamma$ at time $k$ (set $x_{-l}=x_{0}$ for $1 \leq l \leq \gamma$ to simplify notation). Denote the whole history at $k$ by $H_{k}\left(=H_{k}^{k}\right)$. Also, the set $\{z \in X: x \preceq z\}$ will be denoted $[x, M]$ (when $X$ is complete we can say $\sup X=M)$.

Say that a function $f$ on a Euclidean space is strictly increasing if $x \prec \prec y$ implies $f(x) \prec f(y)$. Also, a function $g: T \rightarrow X$ is nowhere weakly increasing over an interval $[\underline{t}, \bar{t}]$ if $t, t^{\prime} \in[\underline{t}, \bar{t}]$ and $t \prec t^{\prime}$ implies $g(x) \npreceq g\left(x^{\prime}\right)$. Note that this is not just the negation of weakly increasing since it rules out the existence of any subinterval of $[\underline{t}, \bar{t}]$ over which the function is increasing.

\footnotetext{
${ }^{6}$ Note that when $\phi$ is a function, i.e. single valued, both concepts coincide with the usual notion of "monotone nondecreasing". Note that if $\phi$ is monotone in the strong set order (i.e. if $x \prec x^{\prime}$ then $\phi(x) \leq_{s} \phi\left(x^{\prime}\right)$ ) then it is weakly increasing and that if $\phi$ is strongly increasing then it is monotone in the strong set order.
} 


\subsection{Games of Strategic Complementarities}

The definition of games of strategic complementarities used here is taken from Milgrom and Shannon (1994) and it is an ordinal generalization of the class of supermodular games introduced by Topkis (1979). Games of strategic complementarities are very common in economics, examples can be found in e.g. Milgrom and Roberts (1990) and Topkis (1998).

Definition 1 Let $N$ be the set of players, each described by a strategy set $S_{i}$ and payoff function $u_{i}: S=\times_{j \in N} S_{j} \rightarrow \mathbf{R}$. A game of (ordinal) strategic complementarities is a pair $\Gamma=\left(N,\left\{\left(S_{i}, u_{i}\right): i \in N\right\}\right)$ where for all $i \in N$ :

1. $S_{i}$ is a complete lattice, $u_{i}$ is quasisupermodular in $s_{i}$ for all $s_{-i} \in S_{-i}$ and satisfies the single-crossing property in $\left(s_{i}, s_{-i}\right)$.

2. The map $s_{-i} \mapsto u_{i}\left(s_{i}, s_{-i}\right)$ is continuous for all $s_{i} \in S_{i}$. Either $s_{i} \mapsto u_{i}\left(s_{i}, s_{-i}\right)$ is continuous for all $s_{-i} \in S_{-i}$ or $s_{i} \mapsto u_{i}\left(s_{i}, s_{-i}\right)$ is upper semicontinuous for all $s_{-i} \in S_{-i}$ and the order interval topology on $S_{i}$ regular. ${ }^{7}$

For all $i \in N$, define $i$ 's best response correspondence $\beta_{i}: S_{-i} \rightarrow S_{i}$ by $\beta_{i}\left(s_{-i}\right)=\operatorname{argmax}_{\hat{s}_{i} \in S_{i}} u_{i}\left(\hat{s}_{i}, s_{-i}\right)$, and the aggregate best response correspondence $\beta: S \rightarrow S$ by $\beta(s)=\times_{i \in N} \beta_{i}\left(s_{-i}\right)$

Definition 2 Let $T$ be a partially ordered set. An indexed family of games of strategic complementarities $(\Gamma(t), t \in T)$ with $\Gamma(t)=\left(N,\left\{\left(S_{i t}, u_{i t}\right): i \in N\right\}\right)$ is an increasing family of games if for all $i \in N, t \mapsto S_{i t}$ is nondecreasing in the strong set order and $u_{i t}$ satisfies the strict single crossing property in $\left(s_{i}, t\right)$ for all $s_{-i} \in S_{-i}$.

Lemma 1 establishes that the results in the paper are applicable to increasing families of games. (Recall that the set of fixed points of the best response correspondence is the set of Nash equilibria of the game from which it was derived). The Lemma follows immediately from known results on comparative statics, although the result when payoffs are only upper semicontinuous requires an additional argument (see Section 8). ${ }^{8}$

\footnotetext{
${ }^{7}$ In particular, if the $S_{i}$ are subsets of a Euclidean space the results apply for upper semicontinuous functions.

${ }^{8}$ Lemma 1 and Theorem 3 imply that $\mathcal{E}(t)$ is nonempty. In this sense, the result is related to Vives's (1990) Theorem 5.1 and to Topkis's (1979) results on algorithms for finding equilibria. It is slightly more general, though, since it relaxes continuity of payoffs to semicontinuity (important in economics for Bertrand-like situations) and requires only quasisupermodularity.
} 
Lemma 1 Let $(\Gamma(t), t \in T)$ be an increasing family of games. Then $\left(\beta_{t}: t \in T\right)$ is an increasing family of uhc correspondences.

\section{Adaptive Dynamics and Comparative Statics}

This section presents results on the dynamic behavior of a system that is perturbed "upwards". The purpose is to capture in a general framework the intuition in the Introduction for games of strategic complementarities: An increase in a parameter makes each player desire larger actions. In turn, because of complementarity between players' choices, the fact that other players pick larger actions goes in the same direction, again making larger choices more desirable. Theorem 1 is the main result. It provides the comparative statics conclusion that the state $x$ of a system that is perturbed upwards is permanently larger. ${ }^{9}$

The (reduced form of the) model will be a correspondence $\phi$ on a lattice. The main application of the results in the paper is to games, where $\phi$ is the aggregate best response correspondence ( $\beta$ in the example in the Introduction). The hypothesis that $x \preceq \inf \phi(x)$ in the theorem is meant to capture an "upward" shift in the state of a system. Typically, $\phi$ will be indexed by partially ordered parameters $t$ so that $\phi_{t}$ is strongly increasing in $t$. This is the case in the example in the Introduction. In this case, the hypothesis is satisfied, since if $x \in \phi_{t}(x)$ is a $t$-equilibrium and $t \prec t^{\prime}$ then $\phi_{t}(x) \preceq \phi_{t^{\prime}}(x)$ strongly implying that $x \preceq \inf \phi_{t^{\prime}}(x)$.

The second hypothesis of the theorem is that dynamics $\left\{x_{k}\right\}$ satisfy $x=x_{0}$ and $\inf \phi\left(\inf H_{k}\right) \preceq$ $x_{k}$ for all $k \geq 1$. Consider a Bertrand oligopoly game and let $\phi$ be its aggregate best response correspondence. The requirement in the theorem is that firms choose prices that are larger than the smallest prices they would consider optimal if they conjecture that all other firms choose their lowest prices in the history of play. Below I argue that most adaptive learning processes that are usually considered satisfy this hypothesis.

Theorem 1 Let $X$ be a lattice and $x \in X$. Let $\phi: X \rightarrow X$ be a correspondence that is weakly increasing on $[x, M]$. If $x \preceq \inf \phi(x)$ then $x$ is a lower bound on any sequence $\left\{x_{k}\right\}$ in $X$ satisfying $x=x_{0}$ and $\inf \phi\left(\inf H_{k}\right) \preceq x_{k}$ for all $k \geq 1$.

\footnotetext{
${ }^{9}$ In the learning literature one of the main questions is that of convergence to equilibria for any starting point. Here the focus lies in the comparison of the starting point and the future behavior of the dynamic process.
} 
Proof: I will show by induction that $x$ is a lower bound on $H_{k}$ for all $k$, which proves the theorem. First, since $\{x\}=H_{1}$ the statement is true for $k=1$. Second, if $x$ is a lower bound on $H_{k-1}$, then inf $H_{k-1} \in[x, M]$. Since $\phi$ is weakly increasing in this interval, $\underline{\phi}(x) \preceq \underline{\phi}\left(\inf H_{k-1}\right)$. Now, $x \preceq \underline{\phi}(x)$ and $\underline{\phi}\left(\inf H_{k-1}\right) \preceq x_{k}$ imply that $x \preceq x_{k}$. Since $H_{k}=H_{k-1} \cup\left\{x_{k}\right\}$, the inductive hypothesis and $x \preceq x_{k}$ imply that $x$ is a lower bound on $H_{k}$.

It should be emphasized that Theorem 1 requires no assumptions of continuity or compactness. It is a simple fact about the order structure of the problem. Correspondingly, there is no implication for convergence to equilibria beyond the remark that if play converges it has to be to a point that is larger than $x$. This is analogous to the literature on comparative statics for decision problems where the results are established independently of the topological assumptions usually needed to guarantee existence. Section 3.1 discusses an important class of adjustment dynamics that satisfy the conditions in Theorem 1 and where conclusions about equilibria can be obtained.

When $\phi$ is interpreted as a best response correspondence, purely adaptive examples of learning processes that satisfy the requirements in the theorem include Cournot best response dynamics, fictitious play (as best response to historic frequency of play) and the "generalized adaptive dynamics" discussed in next subsection. When player's choices are one dimensional then also local "better response" dynamics like gradient optimization algorithms are included.

The backward looking behavior implicit in adaptive play may seem too naive. The hypotheses of Theorem 1 also allows forward looking behavior, though. In the applications to games, any finite number of rounds of "I know that you know ... that I play a best response to $H_{k}$ " will satisfy the condition in Theorem 1 since these rounds are just iterations of $\phi$ (see Milgrom and Roberts (1991) for more on this).

\subsection{Limit Behavior and Equilibria}

The dynamics used here are a specialization of the learning process studied in Milgrom and Roberts $(1990,1991)$ and Milgrom and Shannon (1994). In the context of games, MR's dynamics ask that for any round of play $K$ there be a period $K^{\prime}$ such that if $K^{\prime} \leq k$ then play at round $k$ can be roughly justified as a response to the history of play between $K$ and $k$. MR's class 
of dynamics contains some learning processes that turn out not to yield good predictions for comparative statics. The reason is that they do not necessarily specify behavior for finite sets of rounds of play. Think of $\phi$ as a best response correspondence. In this case, MR's definition basically asks the player to eventually play something moderately rational. This does not rule out that, for example, in the first rounds play goes to both $\inf X$ and $\sup X$, thus destroying the effect of starting at an equilibrium for an "old" parameter value. The idea in the present comparative statics results is that a particular equilibrium is being played before a change in the parameter and that this starting point will influence the situation after an increase in the parameter. The "generalized adaptive dynamics" that I use here retain the flavor in MR's definition while pinning down play in every round.

Definition 3 Let $X$ be a lattice and $\phi: X \rightarrow X$ a correspondence. A sequence $\left\{x_{k}\right\}$ in $X$ is called generalized adaptive dynamics from $\phi$ if there is some $\gamma \in \mathbf{N}$ such that $x_{k} \in$ $\left[\underline{\phi}\left(\inf H_{k}^{\gamma}\right), \bar{\phi}\left(\sup H_{k}^{\gamma}\right)\right]$ for all $k \geq 1$. Let $\mathcal{D}\left(x_{0}, \phi\right)$ be the set of all sequences that are generalized adaptive dynamics from $\phi$ starting at $x_{0}$.

An important example of generalized adaptive dynamics is the set of simple adaptive dynamics from $\phi$ starting at $x \in X$ :

$$
\mathcal{A}(x, \phi)=\left\{\left\{x_{k}\right\}_{k=0}^{\infty}: x_{0}=x, x_{k} \in \phi\left(x_{k-1}\right), k \geq 1\right\} \subset \mathcal{D}(x, \phi) .
$$

These members of the class of adaptive dynamics take the form of Cournot best response dynamics in games and tatonnement price adjustment in market models.

As an illustration consider a Bertrand pricing game with three firms, and let $\gamma=2$. If firms 1 and 2 have set prices $(1,3)$ and $(3,1)$ in the last two periods then firm 3 is "allowed" to set any price between its smallest best response to $(1,1)$ and its largest best response to $(3,3)$. While fictitious play of the kind where the influence of past play smoothly fades away is not included it is easily seen that by allowing $\gamma$ to be large enough any such play can be approximated by generalized adaptive dynamics.

The generalized adaptive dynamics are a restriction of MR's learning process. The main specialization is that I restrict "play" in every time period. A less important difference with 
MR is that the present dynamics incorporates explicitly a bound on the memory of the process. This bound is also present in Milgrom and Roberts's definition (although it is not uniform). ${ }^{10}$ I will denote the set of limits of adaptive dynamics starting at $x \in X$ by

$$
F(x, \phi)=\left\{z \in X: \exists\left\{x_{k}\right\} \in \mathcal{D}(x, \phi) \text { s.t. } z=\lim _{k} x_{k}\right\}
$$

Theorems 2 and 3 are results for uhc correspondences. The main applications are to continuous functions and best response correspondences arising from games of strategic complementarities (see Section 2.3). ${ }^{11}$

Theorem 2 Let $X$ be a complete lattice and $x \in X$. Let $\phi: X \rightarrow X$ be an uhc correspondence that is weakly increasing on $[x, M]$. If $x \preceq \inf \phi(x)$ then $F(x, \phi)$ is nonempty with smallest element $\inf F(x, \phi)=\inf \{z \in \mathcal{E}: x \preceq z\}$ and $\inf F(x, \phi) \in \mathcal{E}$.

Theorem 2 presents the main comparative statics result in this section. If $x \preceq \inf \phi(x)$ then the limits of adaptive dynamics starting at $x$ are larger than the smallest of the equilibria that are larger than $x$. This means that all equilibria reached from $x$ are larger than $x$, a comparative statics statement.

Theorem 2 implies that $\{z \in \mathcal{E}: x \preceq z\}$ is nonempty. This fact yields a proof of MR's comparative statics result for extremal equilibria (and of Milgrom and Shannon's (1994) generalization to games of strategic complementarities, see section 2.3).

Corollary 1 (Milgrom and Roberts (1990), Milgrom and Shannon (1994)) Let $\left(\phi_{t}, t \in\right.$ $T)$ be an increasing family of uhc correspondences. Let $t, t^{\prime} \in T$ such that $t \prec t^{\prime}$. Then $\inf \mathcal{E}(t) \preceq \inf \mathcal{E}\left(t^{\prime}\right)$ and $\sup \mathcal{E}(t) \preceq \sup \mathcal{E}\left(t^{\prime}\right)$.

Proof: The suprema and infima are all well defined since by Zhou's (1994) fixed point theorem the set of equilibria is a complete lattice. Let $e=\sup \mathcal{E}(t)$. Then, by Theorem 2,

\footnotetext{
${ }^{10} \mathrm{My}$ definition is phrased in terms of best responses to historical play instead of MR's definition as strategies that are not dominated when players are restricted to an interval formed from past play. By Lemma 1 of MR this is without loss of generality for supermodular games.

${ }^{11}$ Villas-Boas (1997) presents results on comparative statics of fixed points for equilibrium problems more general than games of strategic complements. Theorem 2, however, does apply to hypotheses similar to, though stronger than, Villas-Boas's (1997).
} 
$e \preceq \inf F\left(e, \phi_{t^{\prime}}\right) \preceq \sup \mathcal{E}\left(t^{\prime}\right)$ since $\inf F\left(e, \phi_{t^{\prime}}\right) \in \mathcal{E}\left(t^{\prime}\right)$. The result for infima follows analogously.

Theorem 3 presents important additional information about the map $x \mapsto F(x, \phi)$. This theorem also turns out to be instrumental in obtaining other results in the paper.

Theorem 3 Let $X$ be a complete lattice and $x \in X$. Let $\phi: X \rightarrow X$ be an uhc correspondence that is weakly increasing on $[x, M]$. If $x \preceq \inf \phi(x)$ then

1. $F(x, \phi)$ has a largest element $\sup F(x, \phi) \in \mathcal{E}$ and for all $\left\{x_{k}\right\} \in \mathcal{D}(x, \phi), \inf F(x, \phi) \preceq$ $\liminf _{k} x_{k} \preceq \lim \sup _{k} x_{k} \preceq \sup F(x, \phi) ;$

2. if, in addition, $\phi$ is strongly increasing over $[x, M]$ then for all $\left\{x_{k}\right\} \in \mathcal{A}(x, \phi), \lim x_{k}$ exists and $\lim x_{k} \in F(x, \phi) \cap \mathcal{E}$.

Theorem 3 is useful for obtaining results for learning processes that converge in distribution, a very common situation in the learning literature. Corollary 2 gives the comparative statics implications of weak convergence when it is achieved. Define the empirical distributions as measures $\mu_{n}$ on the Borel sets of $S$ by $\mu_{n}(O)=\left|\left\{k \leq n: x_{k} \in O\right\}\right| / n$. Clearly an analogous result for convergence of marginal distributions (as in some learning models) is true by using similar arguments.

Corollary 2 Let $\left\{x_{k}\right\}$ be any generalized adaptive play satisfying the conditions of Theorem 2. If the corresponding sequence of empirical distributions $\left\{\mu_{n}\right\}$ converges weakly to $\mu$, then the support of $\mu$ (when the support exists) is contained in $[\inf F(e, \phi), \sup F(e, \phi)]$.

\section{The Correspondence Principle}

\subsection{Non-monotone comparative statics select unstable equilibria}

The main result of this section is Theorem 4: a continuous selector $e(t) \in \mathcal{E}(t)$ that is not monotone increasing must be picking unstable equilibria. The result is established at the same level of generality as Theorem 1. The intuition is very simple. If, in any neighborhood of $e(t)$ there is $e\left(t^{\prime}\right)$ with $t \prec t^{\prime}$ and $e(t) \npreceq e\left(t^{\prime}\right)$, then, starting the $\phi_{t}$-dynamics at $e\left(t^{\prime}\right)$ Theorem 1 
says that play is bounded above by $e\left(t^{\prime}\right)$ and hence cannot converge to $e(t)$. This implies that $e(t)$ is unstable. ${ }^{12}$

This paper uses "asymptotic stability" as a notion of stability; the definition requires that after any small deviation the system converges back to the equilibrium point. This seems an appropriate requirement for an equilibrium when viewed as the steady state of a dynamic system, an equilibrium that is not stable is not likely to be played for a long period of time.

The correspondence $\phi$ here defines a class of dynamics, as opposed to the unique trajectories generated in the dynamical systems that are normally studied. Stability could depend on how a selection of values of $\phi$ is taken, so there is some ambiguity in the usual notions of stability. The definitions below capture this ambiguity.

Definition 4 Let $\phi: X \rightarrow X$. A point $\hat{x} \in X$ is best case stable if there is a neighborhood $V$ of $\hat{x}$ in $X$ such that for all $x$ in $V$, there is a sequence $\left\{x_{k}\right\} \in \mathcal{D}(x, \phi)$ with $x_{k} \rightarrow \hat{x}$. A point $\hat{x} \in X$ is worst case stable if there is a neighborhood $V$ of $\hat{x}$ in $X$ such that for all $x$ in $V$ and all sequences $\left\{x_{k}\right\} \in \mathcal{D}(x, \phi), x_{k} \rightarrow \hat{x}$.

The results in this paper give the strongest possible conclusions: "wrong" comparative statics choose equilibria that are not even best case stable while "correct" comparative statics select worst case stable equilibria.

Theorem 4 Let $\left(\phi_{t}, t \in T\right)$ be an increasing family of correspondences on a lattice $X$ and $T \subset \mathbf{R}^{n}$ be convex. Let $e: T \rightarrow X$ be a continuous selection from $(\mathcal{E}(t): t \in T)$. If e is nowhere weakly increasing over some interval $[\underline{t}, \bar{t}]$ in $T$ then, for all $t \in[\underline{t}, \bar{t}]$ with $\underline{t} \prec t \prec \bar{t}, e(t)$ is not best case stable for $\phi_{t}{ }^{13}$

Proof: Let $t \in[\underline{t}, \bar{t}]$ with $\underline{t} \prec t \prec \bar{t}$ and let $V$ be a neighborhood of $e(t)$. Choose $\hat{t} \in$ $e^{-1}(V) \cap[\underline{t}, \bar{t}]$ with $\hat{t} \prec t$, so $e(\hat{t}) \npreceq e(t)$. Then $e(\hat{t}) \in V$. Let $\left\{x_{k}\right\} \in \mathcal{D}\left(e(\hat{t}), \phi_{t}\right)$. Now, $e(\hat{t}) \in \phi_{\hat{t}}(e(\hat{t}))$ and $\hat{t} \prec t$ so $e(\hat{t}) \preceq \inf \phi_{t}(e(\hat{t}))$. By Theorem $1, e(\hat{t})$ is a lower bound on $\left\{x_{k}\right\}$,

\footnotetext{
${ }^{12}$ I do not discuss the existence of continuous selections, this is also in line with the literature, see the comment in page 9 .

${ }^{13}$ Actually, the proof shows that for any neighborhood $V$ of $e(t)$, there is $x_{0} \in V$ such that $e(t)$ is not an accumulation point for any generalized adaptive dynamics from $\phi_{t}$ starting at $x_{0}$. In particular, if play converges in distribution, $e(t)$ does not belong to the support of the limiting distribution (see Corollary 2).
} 
so any accumulation point $\alpha$ of $\left\{x_{k}\right\}$ satisfies $e(\hat{t}) \preceq \alpha$. Then $e(\hat{t}) \npreceq e(t)$, implies $\alpha \neq e(t)$. In particular $x_{k} \nrightarrow e(t)$.

Remark 1) Theorem 4 applies when elements of $\{e(t): t \in[\underline{t}, \bar{t}]\}$ are not ordered. 2) Since $\mathcal{A}\left(e(t), \phi_{t}\right) \subset \mathcal{D}\left(e(t), \phi_{t}\right)$, non increasing selections are not best case stable when restricting dynamics to $\mathcal{A}\left(e(t), \phi_{t}\right)$. 3) It can be seen from the proof that the theorem is true also allowing dynamics that only satisfy the condition in Theorem 1 .

Note that the source of the instability comes from perturbations in the parameters. This is an additional reason to discard non-increasing selections: consider the example in the Introduction, if workers are slightly wrong about their productivity then by following adjusting dynamics they would move far away from the equilibrium prescribed by the selection.

The main disadvantage of Theorem 4 is that it focuses on continuous selections and convex parameter spaces in $\mathbf{R}^{n}$. In Figure 1 the closest smaller new equilibrium is unstable but we are comparing two discrete parameter values $t \prec t^{\prime}$, not considering continuous selections. ${ }^{14}$ Theorem 5 establishes that the largest new equilibrium that is smaller than the original equilibrium must be unstable, without requiring continuous selections.

Theorem 5 Let $X \subset \mathbf{R}^{m}, T$ a partially ordered set and $\left(f_{t}, t \in T\right)$ be an increasing family of uhc functions. ${ }^{15}$ Fix $t, t^{\prime} \in T$ with $t \prec t^{\prime}$, let $\underline{e}, \bar{e} \in \mathcal{E}\left(t^{\prime}\right)$ with $\underline{e} \prec \bar{e}$ and $\mathcal{E}\left(t^{\prime}\right) \cap[\underline{e}, \bar{e}]=\{\underline{e}, \bar{e}\}$. If $e \in \mathcal{E}(t)$ with $\underline{e} \prec e \prec \bar{e}$ then $\underline{e}$ is not best case stable.

Figure 2 explains the statement of Theorem 5 . In part a) $e \in \mathcal{E}(t)$ is a $t$-equilibrium, $\underline{e}$ is the largest $t^{\prime}$-equilibrium that is smaller than $e$ and $\bar{e}$ is the smallest $t^{\prime}$-equilibrium that is larger than $e$. Then, if there are no $t^{\prime}$-equilibria in $[\underline{e}, \bar{e}]$ (i.e. if there are no equilibria unordered with $e$ in $[\underline{e}, \bar{e}]$ ) then $\underline{e}$ is unstable (and under some additional requirements $\bar{e}$ is stable, see Theorem 7 ). On the other hand, in part b) there are $e_{1}, e_{2}, e_{3} \in \mathcal{E}\left(t^{\prime}\right) \cap[\underline{e}, \bar{e}]$. Then the equilibria $e_{1}, e_{2}$ and $e_{3}$ must be unstable but we do not know if $\underline{e}$ is stable or not. ${ }^{16}$

\footnotetext{
${ }^{14}$ More importantly, it is possible to draw pictures so that the two equilibria cannot be joined by a continuous selection of fixed points.

${ }^{15}$ The result is true when $X$ is a subset of a Banach lattice whose positive cone has nonempty interior, the proof uses only this structure. Moreover, the result is true when only $f_{t^{\prime}}$ is monotone and $e \preceq f\left(e, t^{\prime}\right)$ i.e. the situation in Theorems 1 and 2. I prefer to present this statement to facilitate comparison with Theorems 4 and 6 .

${ }^{16}$ This statement does not follow from Theorem 5 but it is easily seen from its proof why it is true.
} 


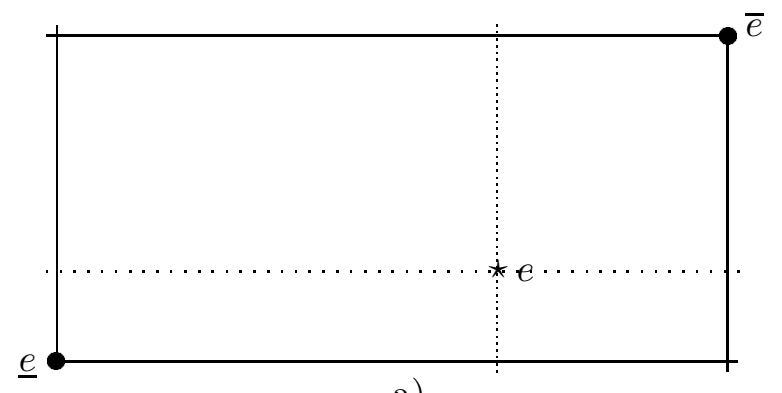

a)

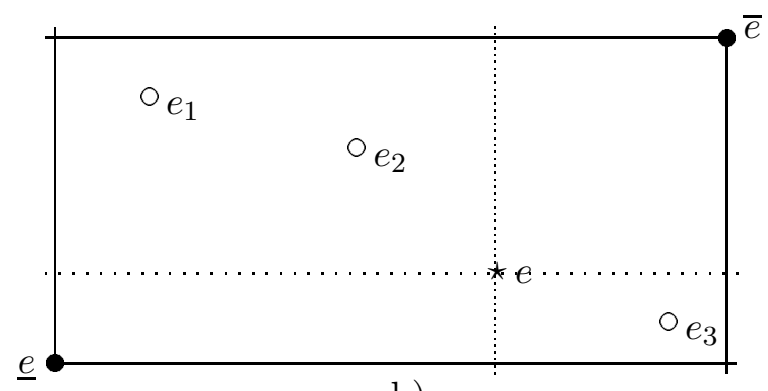

b)

Figure 2: Comparative Statics without continuous selectors

Theorem 5 explains exactly the situation in Figure 1 where stability yields a comparative statics conclusion without the need to restrict to continuous selectors: In a one-dimensional problem like the one in Figure 1 there are no unordered elements. For completeness I present this statement formally in Corollary 3.

Corollary 3 Let $X \subset \mathbf{R}, T$ a partially ordered set and $\left(f_{t}, t \in T\right)$ be an increasing family of uhc functions. Fix $t, t^{\prime} \in T$ with $t \prec t^{\prime}$. If $e \in \mathcal{E}(t), e \notin \mathcal{E}\left(t^{\prime}\right)$ and $\underline{e}$ is the largest $z \in \mathcal{E}\left(t^{\prime}\right)$ with $z \prec e$, then $\underline{e}$ is not best case stable.

Example (The CP and the Monotone Selection Theorem)

The CP can be linked to comparative statics predictions for parameterized optimization problems. This use of the CP is reflected for example in Brock (1983), Hatta (1980) and Magill and Sheinkman (1979). Let $X$ be a lattice, $T$ a partially ordered set and $f: X \times T \rightarrow \mathbf{R}$. Then $\beta: T \rightarrow X$ defined by $\beta(t)=\operatorname{argmax}_{x \in X} f(x, t)$ is a "best response correspondence" that is constant over $X$. Clearly, any $x \in \beta(t)$ is best case stable since for any $\hat{x} \in X$, setting $x_{0}=\hat{x}$ and $x_{k}=x$ for all $k \geq 1$ gives adaptive play that starts at $\hat{x}$ and converges to $x$. Stability means simply that we are at a maximum, hence Samuelson's CP takes the form of the usual requirements for sufficiency of a solution to first order conditions (i.e. concavity and interiority).

By Lemma 1, $(\beta(t): t \in T)$ is an increasing family of correspondences. Here, the conclusion in Theorem 4 is trivial. Any continuous selection of maxima is monotone increasing, but this is already what we know by Milgrom and Shannon's (1994) Monotone Selection Theorem. In the present context one of the applications of the $\mathrm{CP}$ in the older literature is rendered trivial 
by the more recent results on comparative statics. ${ }^{17}$

\subsection{Monotone comparative statics select stable equilibria}

Here I show a converse to the CP: the "right" comparative statics imply stable equilibria. It seems a strong statement to obtain a stability conclusion out of the comparative statics property of the selection of equilibria alone. This comes at the cost of imposing some more structure on the problem: Euclidean spaces, stronger monotonicity assumptions and some regularity conditions on the equilibria.

The main result here, Theorem 6, is a partial converse to Theorem 4. A fixed point $e(t) \in$ $\mathcal{E}(t)$ of the correspondence $\phi_{t}$ is isolated if there is a neighborhood $V$ of $e(t)$ in $X$ such that $V \cap \mathcal{E}(t)=\{e(t)\}$

Theorem 6 Let $X \subset \mathbf{R}^{m}$ and $\left(\phi_{t}, t \in T\right)$ be an increasing family of uhc correspondences with $T \subset \mathbf{R}^{n}$ convex. ${ }^{18}$ Let $e: T \rightarrow X$ be a continuous selection from $(\mathcal{E}(t): t \in T)$. If e is strictly increasing over some interval $[\underline{t}, \bar{t}]$ and $e(t)$ is isolated for some $t \in[\underline{t}, \bar{t}]^{o}$, then $e(t)$ is worst case stable for $\phi_{t}$.

The second result in the section is a partial converse to Theorem 5 . It requires that we rule out some slightly pathological situations.

Definition 5 Given $X \subset \mathbf{R}^{n}$ and a function $f: X \rightarrow X$, say that a fixed point $x$ of $f$ is regular if there is a neighborhood $U$ of $x$ such that

1. $U$ contains no other fixed points of $f$ (i.e. $x$ is locally unique) and

2. the existence of some $y \in U$ with $y \prec \prec f(y) \prec \prec x$ implies that there is also $z \in U$ with $x \prec \prec f(z) \prec \prec z$.

I call the requirement "regularity" because for a function on $\mathbf{R}$ it is the same as asking the fixed point to be regular in the usual sense that its Jacobian has full rank i.e. to cross the

\footnotetext{
${ }^{17}$ Brock (1983), Hatta (1980) and Magill and Sheinkman (1979) did not assume the kind of complementarity required here, so the claim is not that their results are now trivial. Another difference is that these authors related local maxima to local stability, not global as in this paper.

${ }^{18}$ The theorem is true when $X$ is a subset of a Banach lattice whose order cone has a nonempty interior, the proof uses only this structure.
} 

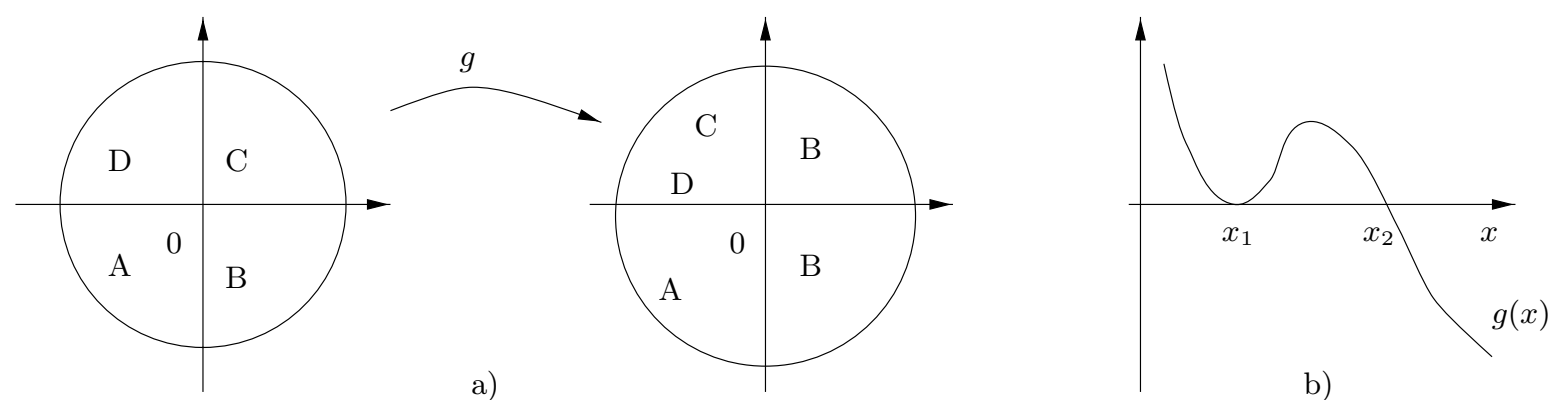

Figure 3: $g(x)=x-f(x)$

diagonal, not only intersect it. This is illustrated in Figure 3, part b). The first fixed point $x_{1}$ of $f$ is not regular because there are smaller points $y$ arbitrarily close so that $y>f(y)$ while the reverse inequality is not true for points close to $x_{1}$ that are larger. On the other hand, it is easy to see that $x_{2}$ is regular both in the usual sense and in accordance with the definition above.

The requirement is in general stronger than the usual notion of regularity, though. For instance, consider Figure 3 a). The map $g: x \mapsto x-f(x)$ in $\mathbf{R}^{2}$ is a counterclockwise smooth rotation of the unit disk that leaves the negative orthant (and 0) fixed but condenses the positive orthant in the $(0,-\infty) \times(0, \infty)$-cone and stretches the $(0, \infty) \times(0,-\infty)$-cone into the positive orthant. I have labeled the intersection of these cones with the disk $A, B, C$ and $D$ so the picture shows the action of the map. Clearly, this transformation can be made as smooth as desired and 0 is a 0 of $g$, i.e. a fixed point of $f$. Then, $g$ is a local diffeomorphism of 0 but 0 is not a regular point in the sense used here because while all elements $y \in A$ satisfy $f(y) \prec \prec y$, for all $z \in B, f(z) \nprec z$.

Theorem 7 Let $X \subset \mathbf{R}^{m}$, $T$ a partially ordered set and $\left(f_{t}, t \in T\right)$ be an increasing family of uhc functions. ${ }^{19}$ Fix $t, t^{\prime} \in T$ with $t \prec t^{\prime}$, let $\underline{e}, \bar{e} \in \mathcal{E}\left(t^{\prime}\right), e \in \mathcal{E}(t)$ with $\underline{e} \prec e \prec \prec \bar{e}$ and $\mathcal{E}\left(t^{\prime}\right) \cap[\underline{e}, \bar{e}]=\{\underline{e}, \bar{e}\}$. Let $f_{t^{\prime}}$ be strictly increasing on $[e, \bar{e}]$. If $e \prec \prec f_{t^{\prime}}(e)$ and $\bar{e}$ is regular, then $\bar{e}$ is worst case stable.

\footnotetext{
${ }^{19}$ Footnote 15 applies.
} 


\section{The Scope of The Correspondence Principle}

Section 4 presents a CP for monotone maps. A question that naturally follows is: does the CP yield comparative statics results for more general fixed point problems? The answer is yes for one-dimensional problems and no for multidimensional problems. The negative result is known in the General Equilibrium literature since at least the early 70s (and motivated Arrow and Hahn (1971) to state that the CP "isn't") and it is indeed related to the ideas in the "anything goes" results of Sonnenschein, Mantel and Debreu (see Mas-Colell, Whinston, and Green (1995) for an exposition).

Proposition 1 considers the one-dimensional case (Milgrom and Roberts (1994) studies mainly this case). The proposition gives a proof of the simple fact that for a function $f$ : $[0,1] \times T \rightarrow[0,1]$ that is increasing in $t$ (but not necessarily in $x$ ), any decreasing selection of equilibria must be picking fixed points where $f$ crosses the identity "from below". This fact is easily seen from picture 4 a). When we consider continuous-time dynamics $\dot{x}_{t}=f\left(x_{t}\right)$ with $f$ continuous or discrete time dynamics $x_{k+1}=f\left(x_{k}\right)$ with $f$ differentiable, crossing from below is enough for instability.

Proposition 1 Let $T \subset \mathbf{R}$ be convex and $f:[0,1] \times T \rightarrow[0,1]$ where $t \mapsto f(x, t)$ is increasing for all $x \in[0,1]$. If $e: T \rightarrow[0,1]$ is a continuous selection of equilibria that is strictly decreasing over some interval $[\underline{t}, \bar{t}]$, then for all $t \in[\underline{t}, \bar{t}]^{\circ}$, there is an $\epsilon>0$ with $f(x, t)<x$ for $x \in$ $(e(t)-\epsilon, e(t))$ and $f(x, t)>x$ for $x \in(e(t), e(t)+\epsilon)$.

Proposition 1 is relevant because it covers most of the traditional uses of the $\mathrm{CP}$, including partial equilibrium analysis and general equilibrium with two goods. Other important examples in the early literature are the macroeconomic IS-LM model and general equilibrium with gross substitutes, which can be obtained from the results on increasing families of correspondences.

Picture $4 \mathrm{~b}$ ) demonstrates that the converse of Proposition 1 (i.e. the analogue of Theorem 6) is not true in the present context. The unique fixed point is not stable (the slope of $f$ is larger in absolute value than 1) but it is increasing in the parameter. Incidentally, note also that this picture rules out comparative statics of the Section 3 form since play starting at an old 


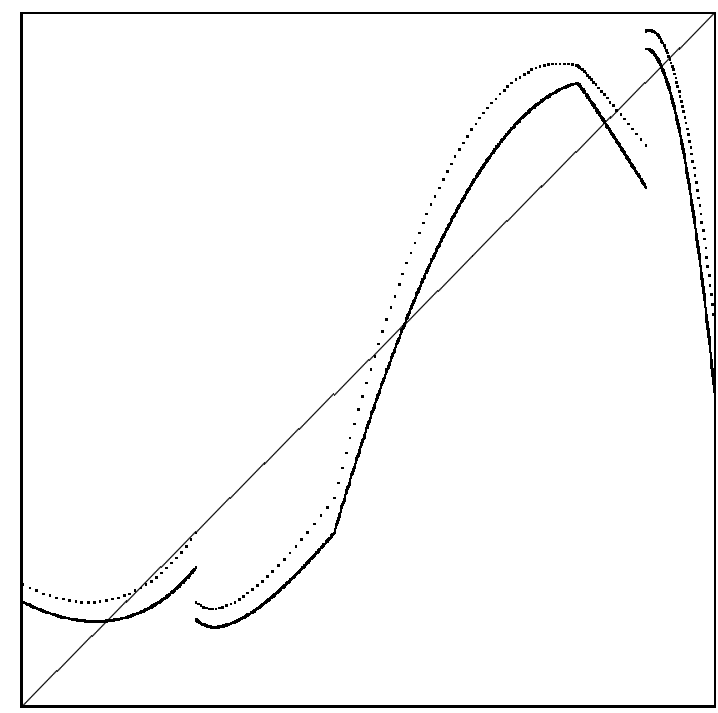

a)

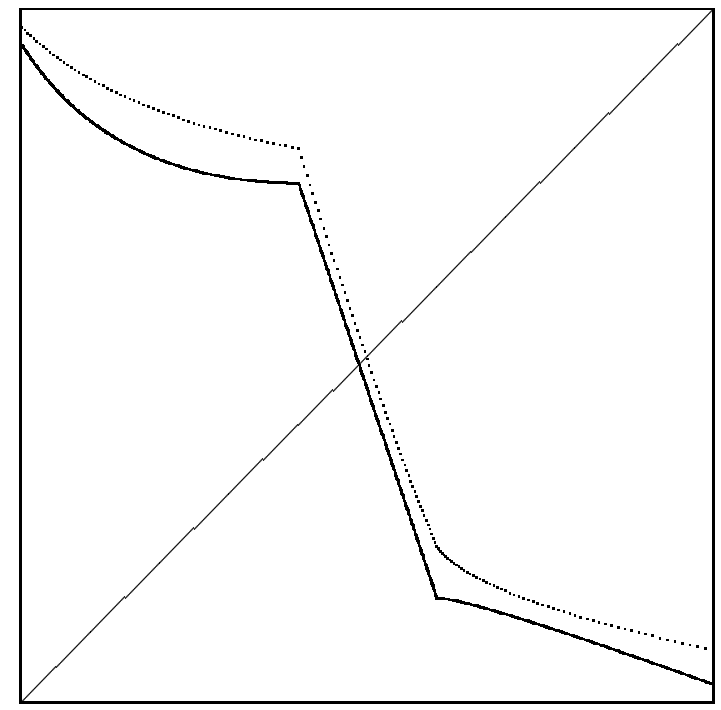

b)

graph of $f\left(., t^{\prime}\right)$

graph of $f(., t)$

Figure 4: An increase in $t$

equilibrium diverges.

Now consider the multidimensional case without the monotonicity that drives the results in the paper. I will give a graphical example that the $\mathrm{CP}$ fails to yield comparative statics predictions. Suggestively, the example is a three good general equilibrium model. ${ }^{20}$ See Arrow and Hahn (1971, p320 and Ch. 10) for a full discussion.

Consider an economy characterized by the excess demand functions $z_{1}, z_{2}$ and $z_{3}$ where $z_{i}: \mathbf{R}^{3} \times[0,1] \rightarrow \mathbf{R}$ is homogeneous of degree 0 , continuous, satisfies Walras's Law and the usual boundary conditions $(i=1,2,3)$. Say that good 1 is the numeraire. The set of equilibria can be seen graphically in Figure 5 as the intersections of the $\left(p_{2}, p_{3}\right)$-loci where $z_{2}$ and $z_{3}$ are zero. Suppose that the map $t \mapsto z_{i}(p, t)$ on $[0,1]$ is increasing for all $p$ and $i=2,3$. Also, let $z_{2}$ and $z_{3}$ be increasing in $p_{2}$ and decreasing in $p_{3}$. By the Sonnenschein-Mantel-Debreu Theorem, there is a well behaved pure exchange economy giving rise to excess demand functions that coincide with $\left(z_{1}, z_{2}, z_{3}\right)$ on a set of prices bounded away from zero.

\footnotetext{
${ }^{20}$ Zeroes of an aggregate demand function $z$ are fixed points of $f(x)=x+z(x)$. Also, note that simple adaptive dynamics correspond to the usual tatonnement adjustment process.
} 


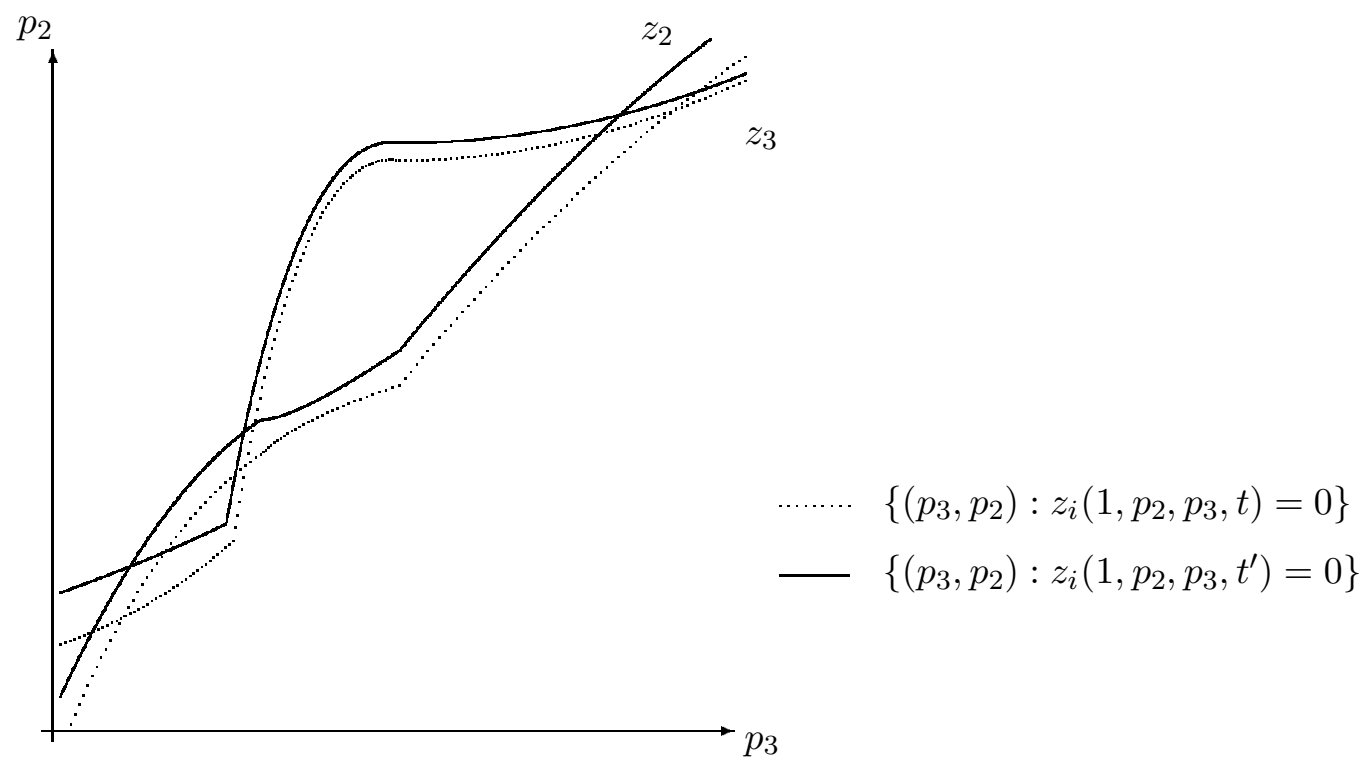

Figure 5: Three good G.E. model

Consider an increase in the parameter $t$. Then, both loci $\left\{\left(p_{3}, p_{2}\right): z_{i}\left(1, p_{2}, p_{3}, t\right)=0\right\}$ must move "down" because, fixing $p_{3}$, a smaller $p_{2}$ is needed to get a zero in $z_{i}$. It is easy to see in the picture that the closest new equilibrium is smaller for the smallest of the fixed points and larger for the largest. But here the two extremal equilibria are stable, hence the CP fails since the requirement of stability and the monotonicity of the excess demand functions in the parameter is not enough to yield comparative statics results.

\section{Applications}

\subsection{Bertrand Oligopoly}

This section shows how the results in the paper are applied to a simple problem: comparative statics of equilibria in the Bertrand oligopoly game. ${ }^{21}$ Assume there is a set $N$ of firms, each facing a continuous demand function $D_{i}\left(p_{i}, p_{-i}\right)$ that depends on its own price $p_{i}$ and the vector of competitors' prices $p_{-i}=\left(p_{1}, \ldots p_{i-1}, p_{i+1}, \ldots p_{n}\right)$. Each firm pays a fixed marginal cost $c_{i}^{t}$ which depends on a parameter $t \in[0,1]$. The vector $\left(c_{1}^{t}, \ldots c_{n}^{t}\right)$ is increasing in $t$.

Let $p_{i} \mapsto D_{i}\left(p_{i}, p_{-i}\right)$ be monotone decreasing and $\left(p_{i}, p_{-i}\right) \mapsto p_{i} D_{i}\left(p_{i}, p_{-i}\right)$ have increasing differences. ${ }^{22}$ The increasing differences condition here is a "substitute products" assumption.

\footnotetext{
${ }^{21}$ This example is also used in MR, Topkis (1979), Milgrom and Shannon (1994) and Topkis (1998).

${ }^{22}$ That cost be linear is not really needed for this to be a game of strategic complements, see Milgrom and Shan-
} 
In this pricing game, firms simultaneously name prices in an interval $[0, \bar{p}]$ of the real line and receive as payoffs their profits $u_{i t}\left(p_{i}, p_{-i}\right)=\left(p_{i}-c_{i}^{t}\right) D_{i}\left(p_{i}, p_{-i}\right)$. It is immediate that $u_{i t}\left(p_{i}, p_{-i}\right)$ satisfies the single crossing property in $\left(p_{i}, p_{-i}\right)$. Trivially $u_{i t}$ is quasisupermodular in $p_{i}$ since $p_{i} \in[0, \bar{p}]$, a chain. Also, since $D_{i}\left(p_{i}, p_{-i}\right)$ is decreasing in $p_{i}, u_{i t}\left(p_{i}, p_{-i}\right)$ satisfies the single crossing condition in $\left(p_{i}, t\right)$. Then, $(\Gamma(t), t \in[0,1])$ with $\Gamma(t)=\left(N,\left\{\left([0, \bar{p}], u_{i t}\right): i \in N\right\}\right)$ is an increasing family of games. Hence, all the results in this paper about the set of Nash equilibrium prices $\mathcal{E}(t)$ hold.

\subsection{Network Externalities}

This section presents an application of the results in the paper to a generalization of Farrell and Saloner's (1985) model of network externalities. Consider a set $N$ of firms that can choose to adopt a new technology in each of $\mathfrak{T}$ rounds of play. There are network externalities in the sense that if a larger number of firms switch to the new standard, then it becomes more profitable for any firm to also change. The model in Farrell and Saloner (1985) has only two firms and two periods but already in this case it is clear that the Implicit Function Theorem is not applicable. Indeed, even with one period, since the choice set is discrete, smoothness and concavity assumptions do not hold. With two stages, the strategy spaces are sets of maps from the history to the switching choice so the calculus based methods are obviously infeasible. Here I allow an arbitrary number of firms (it could be a continuum) and any finite number of periods. ${ }^{23}$

Firm $i$ 's taste for the technology is private information and it is indexed by $\theta_{i} \in \Theta_{i} \subset \mathbf{R}$. Priors on $\Theta=\times_{i \in N} \Theta_{i}$ are given by a Borel probability measure $p$. Final payoffs to firm $i \in N$ will then be given by a bounded measurable function $u_{i}: \Theta_{i} \times\{0,1\}^{N} \rightarrow \mathbf{R}$. Here 1 will represent the choice to switch and 0 to continue using the old technology. I assume that firms are not locked in the new technology once they choose 1: this is only to simplify the notation and it is simple to modify the model to allow for lock in.

Assume that for $a_{i} \in\{0,1\}, a_{-i} \in\{0,1\}^{N \backslash\{i\}}, u_{i}\left(a_{i}, a_{-i}, \theta_{i}\right)$ has increasing differences in non (1994). A sufficient condition for increasing differences to be satisfied is that $D_{i}\left(p_{i}, p_{-i}\right)$ is log-supermodular. In turn, logit, CES and transcendental logarithmic satisfy log-supermodularity, see MR.

${ }^{23}$ This is to simplify, everything can be extended to an infinite horizon or to continuous time. 
$\left(a_{i}, a_{-i}\right)$ and in $\left(a_{i}, \theta_{i}\right) . \quad 24$ The former is the assumption of network externalities while the latter reflects Farrell and Saloner's (1985) assumption that larger types are, other things equal, more likely to switch.

For $\tau=1, \ldots \mathfrak{T}$, let $\mathcal{H}_{\tau}=\{0,1\}^{\tau-1}$ be the set of possible histories until time $\tau$ and $\mathcal{H}=$ $\times_{\tau=1}^{\mathfrak{T}} \mathcal{H}_{\tau}$. Firm $i$ 's strategy space $S_{i}$ is the set of measurable maps $s_{i}: \Theta_{i} \times \mathcal{H} \rightarrow\{0,1\}^{\mathfrak{T}}$, denote by $s_{i}^{\mathfrak{T}}$ its $\mathfrak{T}$-th component. Firm $i$ 's payoff function is

$$
U\left(s_{i}, s_{-i}\right)=\int_{\Theta} u_{i}\left(s_{i}^{\mathfrak{T}}\left(\theta_{i}\right), s_{-i}^{\mathfrak{T}}\left(\theta_{-i}\right), \theta_{i}\right) d p(\theta) .
$$

Proposition $2\left(N,\left\{\left(S_{i}, U_{i}\right): i \in N\right\}\right)$ is a game of strategic complements.

Note that existence of pure strategy equilibria follows by Lemma 1. Typically there will be equilibria with what Farrell and Saloner call "excess inertia": firms switch to the new technology only after several rounds in the game. These are just the non-extremal equilibria of this game. It should also be mentioned that Farrell and Saloner focus on perfect equilibria of this game, these are a subset of the Nash equilibria so the results in the paper apply.

In the network externalities model, the traditional approach based on the Implicit Function Theorem cannot be applied. The equilibria we are usually interested in are not extremal so the existing results on monotone comparative statics of fixed points are not useful.

As an illustration, consider the effect of a reduction in the price of the new technology. Parameterize payoffs by $t \in T \subset \mathbf{R}$ such that for all $i \in N, \theta_{i} \in \Theta_{i}$ and $a_{-i} \in\{0,1\}^{N \backslash\{i\}}$, $u_{i}^{t}\left(1, a_{-i}, \theta_{i}\right)-u_{i}^{t}\left(0, a_{-i}, \theta_{i}\right)$ is increasing in $t$. The interpretation is that an increase in $t$ makes switching cheaper. It is easy to see that the model results in an increasing family of games. Then, reductions in the price of switching set off learning dynamics that involve sequentially higher levels of adoption of the new technology. At the same time, the CP implies that stable selections of equilibria must be monotone increasing.

Another example that cannot be obtained by the existing methods is that an increase in the prior distribution $p$ in the monotone likelihood ratio order would lead to larger equilibria (by Athey's (1996) results, parameterizing by the prior distribution $p$ gives single crossing in $s_{i}$ and the parameter $p$ ).

\footnotetext{
${ }^{24}$ Order any product of $\{0,1\}$ by the componentwise order.
} 


\subsection{Are More Players Good or Bad For Coordination Failures?}

Consider a team of workers that have to choose the effort they put into a common task. Suppose there are complementarities in effort (if one works harder, this makes the others more productive) and positive externalities (if one works harder, this makes the others better off). Then there are typically multiple equilibria with some "hard working equilibria" Pareto dominating other "low effort equilibria". For examples and applications to Macroeconomics, see Cooper and John (1988); other applications include network externalities in Industrial Organization and tax federalism in Public Finance.

These are usually called games with coordination failures. One natural question is whether it is more difficult for a large number of people to "coordinate" on harder effort than a small number.

As shown by Cooper and John, there are two crucial features of coordination failures: strategic complementarities and positive externalities (which they call spillovers). The first is captured by the quasisupermodularity assumption and the second is defined explicitly below. Cooper and John also make assumptions of smoothness of payoffs, strict concavity and Inada conditions to assure that equilibria are interior.

Definition 6 Say that $\Gamma=\left(N,\left\{\left(S_{i}, u_{i}\right): i \in N\right\}\right)$ is a game of positive externalities if it is a game of strategic complementarities and if for all $i, s_{-i} \mapsto u_{i}\left(s_{i}, s_{-i}\right)$ is nondecreasing for all $s_{i} \in S_{i}$

Given a game of positive externalities $\Gamma=\left(N,\left\{\left(S_{i}, u_{i}\right): i \in N\right\}\right)$, let $T=\mathcal{P}(N)$ the power set of $N$ and endow $T$ with the inclusion order, where $t \preceq t^{\prime}$ if and only if $t \subset t^{\prime}$. Add $\left\{0_{i}: i \in N\right\}$ different elements and, for all $i \in N$, set $0_{i} \prec \inf S_{i}$. These will be the "do nothing" choices. Let $f: S \rightarrow \mathbf{R}$ be a (strictly) decreasing function with $\sup \{f(s): s \in S\}<$ 
$\inf \left\{\inf \left\{u_{i}(s): s \in S\right\} i \in N\right\}$ and set ${ }^{25}$

$$
\begin{aligned}
& S_{i t}=\left\{\begin{array}{l}
S_{i} \text { if } i \in t \\
0_{i} \text { if } i \notin t
\end{array}\right. \\
& u_{i t}=\left\{\begin{array}{l}
u_{i} \text { if } i \in t \\
f \text { if } i \notin t
\end{array}\right.
\end{aligned}
$$

The result that more players is Pareto better follows in Proposition 3. These results are in line with Camerer and Knez's (1994) experimental findings for some games of positive externalities. ${ }^{26}$

Proposition 3 For any game of positive externalities $\Gamma(t)$ with a set $t \subset N$ of players and equilibrium $e \in \mathcal{E}(t)$ :

1. If $t \subset t^{\prime} \subset N$ then any convergent general adaptive play starting at $e$, converges to an equilibrium $e^{\prime} \in \mathcal{E}\left(t^{\prime}\right)$ with $e \preceq e^{\prime}$. Moreover, $e^{\prime}$ Pareto dominates e for all players in $t^{\prime}$.

2. Let the strategy spaces be convex subsets of Euclidean spaces and $s_{i} \mapsto u_{i}\left(s_{i}, s_{-i}\right)$ strictly quasiconcave. If $e \in \mathcal{E}(t), e^{\prime} \in \mathcal{E}\left(t^{\prime}\right)$ is the largest equilibrium in $\mathcal{E}\left(t^{\prime}\right)$ that is smaller than $e$ and there are no $t^{\prime}$-equilibria larger than $e^{\prime}$ that are not also larger than $e$, then $e^{\prime}$ is unstable.

Note that the Pareto improvement result is driven by the improvement in the new member's payoffs. If there are strict externalities then also the old members' payoffs increase and in addition we get Pareto domination in part 1 of the Proposition.

\section{Concluding Remarks}

Comparative statics analysis has two uses. One, in "time series" situations, predicts changes in a system before and after a parameter has changed. For example, how do price and quantity in an industry respond after a change in taxes? The second "cross section" use focuses on

\footnotetext{
${ }^{25}$ The choice of "non members" payoffs is just to simplify. If we were to endogenize the option to join this would not be satisfactory.

${ }^{26}$ Camerer and Knez (1994) let subjects play a "weakest link" game (which satisfies the definition of a game of positive externalities) and add players after some rounds of play. This has the effect of worsening the coordination problem. The reason is that in their case, an additional player can only make things worse since their "do nothing" choices are in a sense larger than any strategy of a player in the game. Then, by a simple modification of the present definition and ordering $T=\mathcal{P}(N)$ by set containment, the result follows analogously to Proposition 3 .
} 
differences in two systems that differ in some parameter. For example, if one firm is larger than another, what can we say about the wages that result in a negotiation with their workers?

The results in this paper have a natural application to time series. It is a logical requirement that starting at the old equilibrium, out-of-equilibrium dynamics under the new parameter values give the new prediction.

For cross section questions, this paper makes no predictions. If players sit down to play two games that differ in the value of a parameter, then without information about which equilibria will be selected in general no comparison can be made. One could consider reflecting the ignorance about which equilibrium will be selected by a probability distribution over $\mathcal{E}(t)$. Then, even for monotone problems and using stability as a selection criterion it is easy to generate examples where the endogenous variables can on average be higher or lower after an increase in the parameter. This implies that the usual empirical tests that endogenous and exogenous variables are correlated can be misleading when used on models with multiple equilibria. The problem of cross section comparative statics seems like an important question for future research. ${ }^{27}$.

\section{Proofs}

The following Lemma is a version of MR's Theorem 8 for the present context. It is used here as an auxiliary result in the proof of Theorems 2 and 3. Define iterations of the lower selections from $\phi$ by $\underline{\phi}^{0}(x)=\underline{\phi}(x), \underline{\phi}^{n}(x)=\underline{\phi}\left(\underline{\phi}^{n-1}(x)\right)$ for all $x$ and $n \geq 1$. Define iterations of the upper selections $\bar{\phi}^{n}(x)$ similarly.

Lemma 2 Let $X$ be a complete lattice and $\phi: X \rightarrow X$. Fix $x \in X$ and $\left\{x_{k}\right\} \in \mathcal{D}(x, \phi)$. If $x \preceq \inf \phi(x)$ and $\phi$ is a uhc correspondence that is weakly increasing on $[x, M]$, then, for all $n \in \mathbf{N}$, there exists $K_{n} \in \mathbf{N}$ such that $k \geq K_{n}$ implies $x_{k} \in\left[\underline{\phi}^{n}(x), \bar{\phi}^{n}(M)\right]$.

Proof: The proof proceeds by induction on $n$. To get the result for $n=0$, do induction on $k$ using $K_{0}=1$ : First, note that $H_{1}^{\gamma}=\left\{x_{0}\right\}$ and $x_{0}=x$ so $x_{1} \in\left[\phi\left(\inf H_{1}^{\gamma}\right), \bar{\phi}\left(\sup H_{1}^{\gamma}\right)\right]$

\footnotetext{
${ }^{27}$ Some advances on comparative statics methods that are free from a theory of equilibrium selection are in Echenique and Sabarwal (2000)
} 
implies that $\underline{\phi}(x) \preceq x_{1} \preceq \bar{\phi}(x)$. Also, by weak monotonicity on $[x, M], \bar{\phi}(x) \preceq \bar{\phi}(M)$ so $x_{1} \in[\underline{\phi}(x), \bar{\phi}(M)]$. Suppose now that $\underline{\phi}(x) \preceq x_{l}$ for all $1 \leq l \leq k-1$, so $\underline{\phi}(x)$ is a lower bound on $H_{k}^{\gamma}$. Then $x \preceq \inf \phi(x)=\underline{\phi}(x) \preceq \inf H_{k}^{\gamma}$. So, inf $H_{k}^{\gamma} \in[x, M]$ and weak monotonicity of $\phi$ gives $\inf \phi(x) \preceq \inf \phi\left(\inf H_{k}^{\gamma}\right)$. But then $\inf \phi\left(\inf H_{k}^{\gamma}\right) \preceq x_{k}$. Also, $M$ is an upper bound on $H_{k}^{\gamma}$ and $x \preceq \inf H_{k}^{\gamma} \preceq \sup H_{k}^{\gamma} \preceq M$. By monotonicity on $[x, M], x_{k} \preceq \bar{\phi}\left(\sup H_{k}^{\gamma}\right)=$ $\sup \phi\left(\sup H_{k}^{\gamma}\right) \preceq \sup \phi(M)=\bar{\phi}(M)$. Hence, $x_{k} \in[\phi(x), \bar{\phi}(M)]$. This establishes the result for $n=0$ with $K_{0}=1$.

Now, let $K_{n-1}$ work for $n-1$ in the statement of the Lemma. Set $K_{n}=K_{n-1}+\gamma$. Pick any $k \geq K_{n}$. By the inductive hypothesis, for any $x_{l} \in H_{k}^{\gamma}, \underline{\phi}^{n-1}(x) \preceq x_{l}$. Thus $\underline{\phi}^{n-1}(x)$ is a lower bound on $H_{k}^{\gamma}$ so we get $\underline{\phi}^{n-1}(x) \preceq \inf H_{k}^{\gamma}$. This implies that $\inf \phi\left(\underline{\phi}^{n-1}(x)\right) \preceq \inf \phi\left(\inf H_{k}^{\gamma}\right)$ because $\phi$ is weakly increasing on $[x, M]$ (and $x \preceq \underline{\phi}^{n-1}(x)$ by $x \preceq \inf \phi(x) \preceq \underline{\phi}^{n-1}(x)$ ). Thus, $\underline{\phi}^{n}(x) \preceq \underline{\phi}\left(\inf H_{k}^{\gamma}\right) \preceq x_{k}$. Similarly, by the inductive hypothesis, $\bar{\phi}^{n-1}(M)$ is an upper bound on $H_{k}^{\gamma}$ and therefore $\sup \phi\left(\sup H_{k}^{\gamma}\right) \preceq \sup \phi\left(\bar{\phi}^{n-1}(M)\right)$. This gives $x_{k} \preceq \bar{\phi}^{n}(M)$.

Lemma 3 If $\left\{x_{k}\right\}$ is a monotone sequence in a complete lattice $X$ then $\left\{x_{k}\right\}$ is convergent and $\lim _{k} x_{k}=\bigvee_{k} x_{k}{ }^{28}$

Proof: Let $A$ be the range of $\left\{x_{k}\right\}$ and $x^{*}=\sup A=\bigvee_{k} x_{k}$. Note that $x^{*}$ is also the supremum of the range of any subsequence of $\left\{x_{k}\right\}$ since by monotonicity the range of any subsequence has the same set of upper bounds. Let $V$ be any neighborhood of $x^{*}$. The claim is that eventually $x_{k} \in V$ for all $k$. Since $V^{c}$ is closed and the closed order intervals are a sub-basis for the closed sets in the order interval topology, there is a collection $\left\{\cup_{m=1}^{n_{i}}\left[a_{m}^{i}, b_{m}^{i}\right]: i \in I\right\}$ with $V^{c}=\cap_{i \in I} \cup_{m=1}^{n_{i}}\left[a_{m}^{i}, b_{m}^{i}\right]$ (this is without loss of generality since any $a_{m}^{i}$ or $b_{m}^{i}$ may be inf $X$ or $\sup X$ because $X$ is complete). But $x^{*} \notin V^{c}$ so there is $j \in I$ with $x^{*} \notin \cup_{m=1}^{n_{j}}\left[a_{m}^{j}, b_{m}^{j}\right]$. Now, for any $m=1 \ldots n_{j}$ there can only be a finite number of elements of $\left\{x_{k}\right\}$ in $\left[a_{m}^{j}, b_{m}^{j}\right]$. To see this note that if there is a subsequence $\left\{x_{k_{l}}\right\}$ with $a_{m}^{j} \preceq x_{k_{l}} \preceq b_{m}^{j}$ for all $l \in \mathbf{N}$ then $a_{m}^{j} \preceq x_{k_{l}} \preceq x^{*}$ and $b_{m}^{j}$ is an upper bound on the subsequence so $x^{*} \preceq b_{m}^{j}$. Hence $x^{*} \in\left[a_{m}^{j}, b_{m}^{j}\right]$, a contradiction. Since $n_{j}$ is finite, there can only be a finite number of elements of $\left\{x_{k}\right\}$ in

\footnotetext{
${ }^{28}$ The notation $\bigvee_{k} x_{k}$ refers to the supremum of the range of the sequence $\left\{x_{k}\right\}$.
} 
$\cup_{m=1}^{n_{j}}\left[a_{m}^{j}, b_{m}^{j}\right]$. Hence, eventually, $x_{k} \notin \cap_{i \in I} \cup_{m=1}^{n_{i}}\left[a_{m}^{i}, b_{m}^{i}\right]=V^{c}$. Since $V$ was an arbitrary neighborhood, we conclude $x_{k} \rightarrow x^{*}$.

Lemma 4 Let $\Gamma$ be a game of strategic complementarities. Then $\beta$ is a uhc correspondence that is nondecreasing in the strong set order.

Proof: By Milgrom and Shannon's (1994) Theorem 4, $\beta$ is nondecreasing and by their Corollary 2 it is sublattice valued. Upper hemicontinuity and compact valuedness when $u_{i}$ is continuous in $s_{i}$ follows from Berge's maximum theorem (see e.g. Aliprantis and Border's (1994) Theorem 14.30). For the case where I only require upper semicontinuity, I will use Ausubel and Deneckere's (1993) version of the Theorem of the Maximum (their Theorem 2). Fix $i \in N$ and let $\Pi: S_{-i} \rightarrow \mathbf{R}$ be the projection of the epigraph of $x \mapsto u_{i}(x, \lambda)$ onto $\mathbf{R}$. That is, in this case, $\Pi(\lambda)=\left\{y \in \mathbf{R}: y \leq u_{i}(x, \lambda)\right.$ for some $\left.x \in S_{i}\right\}$ for each $\lambda \in S_{-i}$. The requirement in Ausubel and Deneckere's theorem is that $\Pi$ is lower hemicontinuous (lhc). To see that this is the case, let $\left\{\lambda_{\alpha}\right\}$ be a net in $S_{-i}$ with $\lambda_{\alpha} \rightarrow \lambda\left(\in S_{-i}\right)$. Let $x_{\alpha}$ and $\hat{x}$ be such that $u_{i}\left(x_{\alpha}, \lambda_{\alpha}\right)=\max _{x^{\prime} \in S_{i}} u_{i}\left(x^{\prime}, \lambda_{\alpha}\right)$ for all $\alpha$ and $u_{i}(\hat{x}, \lambda)=\max _{x^{\prime} \in S_{i}} u_{i}\left(x^{\prime}, \lambda\right)$ (possible by compactness of $S_{i}$ and upper semicontinuity of $s_{i} \mapsto u_{i}\left(s_{i}, s_{-i}\right)$ for all $\left.s_{-i}\right)$. Then, $\Pi(\lambda)=\left(-\infty, u_{i}(\hat{x}, \lambda)\right]$. Since $S_{i}$ is compact there is a convergent subnet $\left\{x_{\alpha_{\gamma}}\right\}$ with say $x_{\alpha_{\gamma}} \rightarrow \bar{x} \in S_{i}$. Each $x_{\alpha_{\gamma}}$ achieves the maximum, hence $u_{i}\left(x_{\alpha_{\gamma}}, \lambda_{\alpha_{\gamma}}\right) \geq u_{i}\left(\hat{x}, \lambda_{\alpha_{\gamma}}\right)$. By continuity of $\lambda \mapsto u_{i}(x, \lambda)$ :

$$
\liminf _{\gamma} u_{i}\left(x_{\alpha_{\gamma}}, \lambda_{\alpha_{\gamma}}\right) \geq u_{i}(\hat{x}, \lambda)
$$

Dropping to a further subnet that achieves the lim inf (and summarizing indexes by $\eta$ ) we have $\left\{\left(x_{\eta}, \lambda_{\eta}\right)\right\}$ converging (componentwise), $\left(x_{\eta}, \lambda_{\eta}\right) \rightarrow(\bar{x}, \lambda)$ and $u_{i}\left(x_{\eta}, \lambda_{\eta}\right) \rightarrow \zeta \geq u_{i}(\hat{x}, \lambda)$. For any $y \in \Pi(\lambda)$, let $y_{\eta}=u_{i}\left(x_{\eta}, \lambda_{\eta}\right) \wedge y$. Then, $y_{\eta} \in \Pi\left(\lambda_{\eta}\right)$ for all $\eta$ and $y_{\eta} \rightarrow y$ (since $y \leq \zeta$ so when $y=\zeta$ it is immediate and when $y<\zeta$, there is $\eta_{0}$ so that $\eta_{0}<\eta$ implies $u_{i}\left(x_{\eta}, \lambda_{\eta}\right)>(\zeta+y) / 2$ hence $y_{\eta}=y$ for all $\left.\eta_{0}<\eta\right)$.

Thus, given that $\lambda_{\eta}$ is a subnet of $\lambda_{\alpha}$ we have established that $\Pi$ is l.h.c. This satisfies the requirement that $\Pi$ is continuous in the lower Vietoris topology in Ausubel and Deneckere's (1993) theorem. Then, since $S_{i}$ is a regular topological space and $u_{i}$ is upper semicontinuous, $\beta_{i}$ is nonempty, compact-valued and upper hemicontinuous. 
Hence, each $\beta_{i}$ is upper hemicontinuous and compact valued. Then by Theorem 14.27 in Aliprantis and Border (1994), $\beta$ is upper hemicontinuous and compact valued. Thus it is subcomplete by the Frink-Birkhoff characterization that subcompleteness is the same as compactness in the order interval topology (see Birkhoff's (1967)).

Proof of Lemma 1 Given $t \in T, s \mapsto \beta(s, t)$ is increasing in the strong set order by Lemma 4 , hence it is weakly increasing. Given $s \in S$, any selection from $t \mapsto \beta(s, t)$ is increasing by Milgrom and Shannon's (1994) Monotone Selection Theorem, hence it is strongly increasing. Upper-hemi-continuity and sub complete sublattice valuedness is given by Lemma 4 .

Proof of Theorems 2 and 3 Define the sequences $\left\{x_{k}\right\},\left\{y_{k}\right\}$ by $x_{0}=y_{0}=x$ and $x_{k}=\underline{\phi}^{k}(x)$ and $y_{k}=\bar{\phi}^{k}(x)$ for $k \geq 1$ (all infima and suprema are well defined by the completeness of $X$ ). First I will show by induction that $\left\{x_{k}\right\}$ and $\left\{y_{k}\right\}$ are sequences in $[x, M]$. Since $x \preceq \inf \phi(x) \preceq$ $\sup \phi(x), x_{0}=x \preceq x_{1} \preceq y_{1}$. If $x \preceq x_{k-1}$, then $x, x_{k-1} \in[x, M]$. By weak monotonicity of $\phi$ on $[x, M]$, then, $x \preceq \inf \phi(x) \preceq \inf \phi\left(x_{k-1}\right)$. This implies that $x_{k} \in[x, M]$. The argument for $\left\{y_{k}\right\}$ is identical.

Now, $\phi$ is weakly increasing on $[x, M]$ and $x_{0} \preceq x_{1} \preceq y_{1}$, so $x_{k-1} \preceq x_{k}$ and $y_{k-1} \preceq y_{k}$ for all $k$. Thus, $\left\{x_{k}\right\}$ and $\left\{y_{k}\right\}$ are monotone sequences in $X$. By Lemma $3, x_{k} \rightarrow x^{*}=\bigvee_{k} x_{k}$ and $y_{k} \rightarrow y^{*}=\bigvee_{k} y_{k}$

Also, $\phi$ is sub-complete and sublattice valued on $[x, M]$, so $x_{k} \in \phi\left(x_{k-1}\right)$ for all $k \geq 1$ and thus $\left\{x_{k}\right\} \in \mathcal{A}(x, \phi) \subset \mathcal{D}(x, \phi)$. This implies that $x^{*} \in F(x, \phi)$ and thus $F(x, \phi)$ is nonempty. Now, set $z_{k}=x_{k+1} \in \phi\left(x_{k}\right)$ for all $k \geq 1$. Since $x_{k} \rightarrow x^{*}$ and $\phi$ is upper hemicontinuous on $[x, M]$ and closed valued, (see Theorem 14.17 in Aliprantis and Border (1994)) there is $z \in \phi\left(x^{*}\right)$ and a subsequence $\left\{z_{k_{l}}\right\}$ of $\left\{z_{k}\right\}$ such that $z_{k_{l}} \rightarrow z$. But $\left\{z_{k_{l}}\right\}$ is also a subsequence of $\left\{x_{k}\right\}$, and the order interval topology on $X$ is Hausdorff because $X$ is a complete lattice, so $z=x^{*}$. Then $x^{*} \in \phi\left(x^{*}\right)$ so $x^{*} \in \mathcal{E}$. Clearly, $x=x_{0} \preceq x^{*}$ since $x^{*}=\sup A$. The reasoning for $\left\{y_{k}\right\}$ is analogous and gives $y_{k} \rightarrow \bigvee_{k} y_{k} \in F(x, \phi) \cap \mathcal{E}$.

Let $\left\{z_{k}\right\} \in \mathcal{D}(x, \phi)$ and let $\left\{x_{k}\right\}$ and $\left\{y_{k}\right\}$ be defined as above. I will show by induction that $\left\{y_{k}\right\}$ is pointwise larger than $\left\{z_{k}\right\}$. First, $x_{0} \preceq z_{0} \preceq y_{0}$ trivially. If $x_{l} \preceq z_{l} \preceq y_{l}$ for all $1 \leq l \leq k-1$ then $z_{k-1} \in[x, M]$, and $y_{k-1}=\sup \left\{y_{l}: 1 \leq l \leq k-1\right\}$ is an upper bound on $H_{k}^{\gamma}$. 
Then $\sup H_{k}^{\gamma} \preceq y_{k-1}$. By weak monotonicity, then, $z_{k} \preceq \sup \phi\left(\sup H_{k}^{\gamma}\right) \preceq \sup \phi\left(y_{k-1}\right)=y_{k}$. Hence, $\left\{y_{k}\right\}$ is pointwise larger than $\left\{z_{k}\right\}$, which implies that the set of upper bounds of the range of $\left\{y_{k}\right\}$ is contained in the set of upper bounds of $\left\{z_{k}\right\}$. But $y_{k} \rightarrow y^{*}=\bigvee_{k} y_{k}$ so $\lim \sup _{k} z_{k} \preceq y^{*}$. By Lemma 2 , for all $n, x_{n}=\underline{\phi}^{n}(x) \preceq \liminf z_{k}$. Thus $\liminf z_{k}$ is an upper bound on the range of $\left\{x_{n}\right\}$. But $x_{n} \rightarrow \bigvee_{n} x_{n}=x^{*}$, so we get $x^{*} \preceq \liminf z_{k}$. Hence, $x^{*} \preceq \liminf z_{k} \preceq \lim \sup z_{k} \preceq y^{*}$. In particular, if $\left\{z_{k}\right\} \in \mathcal{D}(x, \phi)$ is convergent the corresponding limit will also be in $\left[x^{*}, y^{*}\right]$. Thus, $x^{*}$ and $y^{*}$ are, respectively, lower and upper bounds on $F(x, \phi)$ and since $x^{*}, y^{*} \in F(x, \phi)$ this implies $x^{*}=\inf F(x, \phi)$ and $y^{*}=\sup F(x, \phi)$. Hence $F(x, \phi)$ is nonempty and has a smallest element, $\inf F(x, \phi) \in \mathcal{E}$, and a largest element, $\sup F(x, \phi) \in \mathcal{E}$, proving item 1 in Theorem 3 and the first half of Theorem 2 .

To finish the proof of Theorem 2, first note that $x \preceq \inf F(x, \phi)$ since inf $F(x, \phi)=\bigvee_{k} x_{k}$. Together with $\inf F(x, \phi) \in \mathcal{E}$ this implies that $\{z \in \mathcal{E}: x \preceq z\} \neq \emptyset$. Let $e \in\{z \in \mathcal{E}: x \preceq z\}$. By induction I show that $e$ is an upper bound on the range of $\left\{x_{k}\right\}$. First note that $x_{0}=x \preceq e$ implies $x_{0}, e \in[x, M]$. Then, $x_{k-1} \preceq e$ and weak monotonicity of $\phi$ on $[x, M]$ imply that $x_{k}=\inf \phi\left(x_{k-1}\right) \preceq \inf \phi(e)$ and $x_{k} \in[x, M]$. But $e \in \phi(e)$ so $\inf \phi(e) \preceq e$. Thus, $x_{k} \preceq e$ for all $k$. Since $\inf F(x, \phi)=\bigvee_{k} x_{k}$ this implies that $\inf F(x, \phi)$ is a lower bound on $\{z \in \mathcal{E}: x \preceq z\}$. But we proved that inf $F(x, \phi) \in\{z \in \mathcal{E}: x \preceq z\}$, thus proving Theorem 2 .

Finally, assume that $\phi$ is strongly increasing over $[x, M]$. Let $\left\{z_{k}\right\} \in \mathcal{A}(x, \phi)$. By the argument above, $\left\{z_{k}\right\}$ is a sequence in $[x, M]$ and $z_{0}=x \preceq z_{1}$. Since $\phi$ is strongly increasing, $z_{k-2} \preceq z_{k-1}, z_{k-1} \in \phi\left(z_{k-2}\right)$ and $z_{k} \in \phi\left(z_{k-1}\right)$, we conclude that $z_{k-1} \preceq z_{k}$. Inductively, then, $\left\{z_{k}\right\}$ is monotone. By repeating the argument made above for the infimum selection $\left\{x_{k}\right\}$ we obtain that $\lim _{k} z_{k}$ exists and $\lim _{k} z_{k} \in \mathcal{E}$. Since $\left\{z_{k}\right\} \in \mathcal{A}(x, \phi) \subset \mathcal{D}(x, \phi), \lim _{k} z_{k} \in F(x, \phi)$. This proves item 2 in Theorem 3.

Proof of Corollary 2 Let $\left\{z_{k}\right\} \in \mathcal{D}(x, \phi)$. Define the sequences $\left\{x_{k}\right\}$ and $\left\{y_{k}\right\}$ like in the proof of Theorems 2 and 3 . We already know that $y_{k} \rightarrow \bigvee_{k} y_{k}=y^{*}$ and that $z_{k} \preceq$ $y_{k} \preceq y^{*}$ for all $k$. Let the sequence $\left\{O_{n}\right\}$ be given by $O_{n}=\left[x_{n}, y^{*}\right]^{c}$, which is a Borel set for the interval topology for each $n$. Given $n \in \mathbf{N}$, take $K_{n}$ from Lemma 2. For any $k \geq K_{n}, x_{n} \preceq z_{k}$ so $z_{k} \in\left[x_{n}, y^{*}\right]$. Hence, $\mu_{k}\left(O_{n}\right) \leq K_{n} / k$. Since $\mu_{k} \rightarrow \mu$ weakly, $\mu\left(O_{n}\right)=$ 
$\int \mathbf{1}_{O_{n}} d \mu=\lim _{k \rightarrow \infty} \int \mathbf{1}_{O_{n}} d \mu_{k}=\lim _{k \rightarrow \infty} \mu_{k}\left(O_{n}\right)=0$. But $x_{n} \rightarrow \bigvee_{n} x_{n}=\inf F(x, \phi)$ and $y^{*}=\sup F(x, \phi)$, so it immediately follows that $[\inf F(x, \phi), \sup F(x, \phi)]=\cap_{n}\left[x_{n}, y^{*}\right]$. By the De Morgan laws, $[\inf F(x, \phi), \sup F(x, \phi)]^{c}=\cup_{n}\left[x_{n}, y^{*}\right]^{c}$. Then by "left continuity" of $\mu$, $\mu\left([\inf F(x, \phi), \sup F(x, \phi)]^{c}\right)=\mu\left(\cup_{n} O_{n}\right)=0$.

Proof of Theorem 5 Let $\left\{x_{k}\right\}$ be the only element of $\mathcal{A}\left(e, f_{t^{\prime}}\right)\left(f_{t^{\prime}}\right.$ is a function so $\mathcal{A}\left(e, f_{t^{\prime}}\right)$ is a singleton). By Theorem 3 , since $f_{t^{\prime}}$ is strongly increasing and continuous, $\lim _{k} x_{k}=$ $\inf \left\{z \in \mathcal{E}\left(t^{\prime}\right): e \preceq z\right\}$. Then $\bar{e} \in\left\{z \in \mathcal{E}\left(t^{\prime}\right): e \preceq z\right\}$ and there exist no $z \in \mathcal{E}\left(t^{\prime}\right)$ with $e \preceq z \prec \bar{e}$ because $\mathcal{E}\left(t^{\prime}\right) \cap[\underline{e}, \bar{e}]=\{\underline{e}, \bar{e}\}$. Hence $\bar{e}=\inf \left\{z \in \mathcal{E}\left(t^{\prime}\right): e \preceq z\right\}$ and $\lim x_{k}=\bar{e}$.

Let $P=\left\{x \in \mathbf{R}^{m}: 0 \preceq x\right\}$ be the positive cone in $\mathbf{R}^{m}$. Now, $\bar{e}-e \in P^{o}$ so there exists a neighborhood $W$ of $\bar{e}$ with $e \prec z$ for all $z \in W$. Let $z \in W \cap[\underline{e}, \bar{e}]$ and let $\left\{z_{k}\right\} \in \mathcal{A}\left(z, f\left(., t^{\prime}\right)\right)$. By induction I show that $x_{k} \preceq z_{k} \preceq \bar{e}$ for all $k$. First, $x_{0}=e \prec z_{0} \preceq \bar{e}$. Second, if $x_{k-1} \preceq z_{k-1} \preceq \bar{e}$ then monotonicity of $f_{t^{\prime}}$ implies that $f_{t^{\prime}}\left(x_{k-1}\right) \preceq f_{t^{\prime}}\left(z_{k-1}\right) \preceq f_{t^{\prime}}(\bar{e})=\bar{e}$. Hence, $x_{k} \preceq z_{k} \preceq \bar{e}$ for all $k$. But $\lim _{k} x_{k}=\bar{e}$ so $\bar{e} \preceq \lim \inf z_{k} \preceq \lim \sup z_{k} \preceq \bar{e}$, which then implies that $z_{k} \rightarrow \bar{e}$.

By Dancer and Hess's (1991) Proposition 1, either there exist $z$ in $[\underline{e}, \bar{e}]$ arbitrarily close to $\bar{e}$ such that $f_{t^{\prime}}(z) \prec z$ or there exist $z$ in $[\underline{e}, \bar{e}]$ arbitrarily close to $\underline{e}$ such that $z \prec f_{t^{\prime}}(z)$. The first possibility is ruled out by the preceding paragraph because for any $z$ in that situation $\left\{z_{k}\right\} \in \mathcal{A}\left(z, f_{t^{\prime}}\right)$ would be a monotone decreasing sequence that does not converge to $\bar{e}$.

Hence, for every neighborhood $N$ of $\underline{e}$ there is $z \in N \cap[\underline{e}, \bar{e}]$ with $\underline{e} \prec z \prec f_{t^{\prime}}(z)$. Let $\left\{z_{k}\right\} \in \mathcal{D}\left(z, f_{t^{\prime}}\right)$ and $\left\{\hat{z}_{k}\right\} \in \mathcal{A}\left(z, f_{t^{\prime}}\right)$. Using the fact that $z \preceq \hat{z}_{0}$ and $z \preceq \hat{z}_{k-1}$, we conclude that $z \prec f_{t^{\prime}}(\hat{z}) \preceq f_{t^{\prime}}\left(\hat{z}_{k-1}\right)=\hat{z}_{k}$. Therefore, inductively, $z$ is a lower bound on the range of $\left\{\hat{z}_{k}\right\}$. Hence, $\underline{e} \prec z \preceq \liminf _{k} \hat{z}_{k}$. Here, $\left\{\hat{z}_{k}\right\}$ coincides with the infimum and supremum selection in Theorem 3. Therefore, $\underline{e} \prec z \preceq \liminf _{k} \hat{z}_{k} \preceq \lim \inf _{k} z_{k}$ so and $z_{k} \nrightarrow \underline{e}$ (in fact using Theorem 3 it is easy to see that $\left.z_{k} \rightarrow \bar{e}\right)$. Since $N$ was arbitrary, $\underline{e}$ is not best case stable.

Proof of Theorem 6 Let $t \in[\underline{t}, \bar{t}]^{o}$ be such that $e(t)$ is isolated. Let $N$ be a neighborhood of $e(t)$ with $N \cap \mathcal{E}(t)=\{e(t)\}$ and let $P=\left\{x \in \mathbf{R}^{m}: 0 \preceq x\right\}$ be the positive cone in $\mathbf{R}^{m}$. Let $B_{r}$ and $B_{2 r}$ be open balls contained in $N$ with center $e(t)$ and radii $r$ and $2 r$, respectively. Take $t_{1}, t_{2} \in e^{-1}\left(B_{r}\right) \cap[\underline{t}, \bar{t}]^{o}$ with $t_{1} \prec \prec t \prec \prec t_{2}$. Note then that $\left[e\left(t_{0}\right), e\left(t_{1}\right)\right] \subset B_{2 r}$. To see this, set $e(t)=0$ without loss of generality. If $x \in\left[e\left(t_{0}\right), e\left(t_{1}\right)\right]$ then $x \vee 0 \preceq e\left(t_{1}\right)$ and $(-x) \wedge 0 \preceq-e\left(t_{0}\right)$. 
Then

$|x|=x \vee 0+(-x) \wedge 0 \preceq e\left(t_{1}\right)-e\left(t_{0}\right)=0 \vee\left(e\left(t_{1}\right)+e\left(t_{0}\right)\right)-0 \wedge\left(e\left(t_{1}\right)+e\left(t_{0}\right)\right)=\left|e\left(t_{0}\right)+e\left(t_{1}\right)\right|$

so since $\|$.$\| is a lattice norm, \|x\| \leq\left\|e\left(t_{0}\right)+e\left(t_{1}\right)\right\|$. But $e\left(t_{0}\right), e\left(t_{1}\right) \in B_{r}$ so

$$
\|x\| \leq 2 \max \left\{\left\|e\left(t_{0}\right)\right\|,\left\|e\left(t_{1}\right)\right\|\right\} \leq 2 r
$$

Hence $x \in B_{2 r}$.

Now, $e\left(t_{0}\right) \prec e(t) \prec e\left(t_{1}\right)$, i.e. $e(t) \in e\left(t_{0}\right)+P^{o}$ and $e(t) \in e\left(t_{1}\right)-P^{o}$. Let $V=\left(e\left(t_{0}\right)+\right.$ $\left.P^{o}\right) \cap\left(e\left(t_{1}\right)-P^{o}\right) \cap X$, a $X$-relatively open neighborhood of $e(t)$. The claim is that $V$ satisfies the definition of worst case stability.

Let the sequences $\left\{y_{n}\right\}$ and $\left\{z_{n}\right\}$ be such that $y_{0}=e\left(t_{0}\right), z_{0}=e\left(t_{1}\right)$ and $y_{n}=\inf \phi_{t}\left(y_{n-1}\right)$, $z_{n}=\sup \phi_{t}\left(z_{n-1}\right)$ for all $n \geq 1$. Then $\left\{y_{n}\right\}$ and $\left\{z_{n}\right\}$ are simple adaptive play from $\phi_{t}$ and, by Theorem $3, y_{n} \rightarrow e^{\prime}$ and $z_{n} \rightarrow e^{\prime \prime}$ with $e^{\prime}, e^{\prime \prime} \in \mathcal{E}(t)$. Now, for any $x \in V$, let $\left\{x_{k}\right\}$ be some arbitrary generalized adaptive play from $\phi_{t}$ starting at $x_{0}=x$. By Lemma 2, applied twice (once dually on $X$ to get the second inequality), $y_{n} \preceq \liminf x_{k} x_{k} \preceq \lim \sup _{k} x_{k} \preceq z_{n}$ for all $n$. This implies that $e^{\prime} \preceq \liminf _{k} x_{k} \preceq \lim \sup _{k} x_{k} \preceq e^{\prime \prime}$. Then $e^{\prime}, e^{\prime \prime} \in\left[e\left(t_{0}\right), e\left(t_{1}\right)\right] \subset B \subset N$ and therefore $e^{\prime}=e^{\prime \prime}=e(t)$ by local isolation. Then, $e(t) \preceq \liminf _{k} x_{k} \preceq \lim \sup _{k} x_{k} \preceq e(t)$, so that $x_{k} \rightarrow e(t)$. Thus $V$ satisfies the definition of worst case stability.

Proof of Theorem 7 Let $\left\{\left\{x_{k}\right\}\right\}=\mathcal{A}\left(e, f_{t^{\prime}}\right)$. It is easy to see by induction that $\left\{x_{k}\right\}$ is a strictly increasing sequence since $f_{t^{\prime}}$ is strictly increasing on $[e, \bar{e}]$ and $e \prec \prec f_{t^{\prime}}(e)$ First, $x_{0}=$ $e \prec f_{t^{\prime}}(e)=x_{1}$. Second, if $x_{k-2} \prec x_{k-1}$ then $x_{k-1} \prec x_{k}$. Now, let $U$ be the neighborhood of $\bar{e}$ in the definition of regularity, since $x_{k} \rightarrow \bar{e}$ (see the proof of Theorem 5), there is $K \in \mathbf{N}$ such that $k \geq K$ implies $x_{k} \in U$.

Since $x_{K} \prec \prec x_{K+1}=f_{t^{\prime}}\left(x_{K}\right) \prec \bar{e}$, and $\bar{e}$ is regular there is $y \in U$ with $\bar{e} \prec \prec f_{t^{\prime}}(y) \prec \prec y$. So, $x_{K} \prec \prec \bar{e} \prec \prec y$ and therefore there are open sets $O_{1}$ and $O_{2}$ with $y-\bar{e} \in O_{1} \subset P^{o}$ and $\bar{e}-x_{K} \in$ $O_{2} \subset P^{o}$. Let $O=\left(y-O_{1}\right) \cap\left(x_{K}+O_{2}\right)$, clearly $O$ is an open neighborhood of $\bar{e}$. Let $z \in O$, then $y-z \in P^{o}$ and $z-x_{K} \in P^{o}$ so that $x_{K} \prec \prec z \prec \prec y$. Let $\left\{z_{k}\right\} \in \mathcal{D}\left(z, f_{t^{\prime}}\right)$ and $\left\{y_{k}\right\} \in \mathcal{A}\left(z, f_{t^{\prime}}\right)$, note that $\left\{x_{k}\right\}$ and $\left\{y_{k}\right\}$ coincide with the infimum and supremum selections because $f$ is single valued. Inductively, then, $x_{K+k} \preceq z_{k} \preceq y_{k}$ for all $k \in \mathbf{N}$ because $x_{K} \preceq z_{0} \preceq y$ and if 
$x_{K+k-1} \preceq z_{k-1} \preceq y_{k-1}$, then $f_{t^{\prime}}\left(x_{K}\right) \preceq f_{t^{\prime}}(z) \preceq f_{t^{\prime}}(y)$. By Theorem $3, y_{k} \rightarrow \bar{e}$ so

$$
\bar{e}=\lim _{k} x_{k} \preceq \liminf _{k} z_{k} \preceq \limsup _{k} z_{k} \preceq \lim _{k} y_{k}=\bar{e}
$$

This establishes that $\bar{e}$ is worst case stable.

Proof of Proposition 1 Let $t \in[\underline{t}, \bar{t}]^{o}$. Note that $e\left([\underline{t}, \bar{t}]^{o}\right) \cap\{0,1\}=\emptyset$ or $e$ would not be strictly decreasing. Pick $\epsilon>0$ such that $(e(t)-\epsilon, e(t)+\epsilon) \subset e\left([\underline{t}, \bar{t}]^{o}\right)$ (possible since $e$ is decreasing and continuous). If $x \in(e(t)-\epsilon, e(t))$ then there is $t^{\prime} \in[\underline{t}, \bar{t}]^{o}$ such that $x=e\left(t^{\prime}\right)$.

Now, $t^{\prime} \leq t$ implies that $x=e\left(t^{\prime}\right) \geq e(t)$ so we must have $t^{\prime}>t$. Since $f$ is increasing in $t$ we have $x=f\left(x, t^{\prime}\right)>f(x, t)$. The proof that $x<f(x, t)$ for $x \in(e(t), e(t)+\epsilon)$ is analogous.

Proof of Proposition 2 Clearly $S_{i}$ is a lattice in the componentwise order, by Tychonoff's Theorem it is compact. It is immediate that $U_{i}$ is continuous since a convergent net in $S=$ $\times_{i \in N} S_{i}$ converges in its $\mathfrak{T}$-th component, which by Lebesgue's Dominated Convergence Theorem implies that the integral in the definition of $U_{i}$ converges. To see that $s_{i} \mapsto U\left(s_{i}, s_{-i}\right)$ is quasisupermodular note that for any $s_{i}, s_{i}^{\prime} \in S_{i}$ and $s_{-i} \in S_{-i}, U\left(s_{i}, s_{-i}\right)-U\left(s_{i} \wedge s_{i}^{\prime}, s_{-i}\right)=$

$$
\int_{\left\{\theta_{i}: s_{i}^{\mathfrak{T}}\left(\theta_{i}\right)=1, s_{i}^{\prime \mathfrak{T}}\left(\theta_{i}\right)=0\right\}}\left[u_{i}\left(1, s_{-i}^{\mathbb{T}}\left(\theta_{-i}\right), \theta_{i}\right)-u_{i}\left(0, s_{-i}^{\mathfrak{T}}\left(\theta_{-i}\right), \theta_{i}\right)\right] d p(\theta) .
$$

But this expression is the same as $U\left(s_{i} \vee s_{i}^{\prime}, s_{-i}\right)-U\left(s_{i}^{\prime}, s_{-i}\right)$. Finally, $U_{i}$ satisfies the single crossing property in $\left(s_{i}, s_{-i}\right)$ because $u_{i}$ has increasing differences.

Proof of Proposition 3 The claim is that the family of games $\Gamma(t)=\left(N,\left\{\left(S_{i t}, u_{i t}\right): i \in N\right\}\right)$ is an increasing family of games. Clearly for any $t, \Gamma(t)$ is a game of strategic complementarities and $S_{i t}$ is increasing in $t$. The strict single crossing condition in $(s, t)$ is satisfied vacuously for $u_{i, t}$ when $i \notin t$ because $f$ is monotone decreasing. For $i \in t$, strict single crossing in $(s, t)$ follows by strict single crossing in $\left(s_{i}, s_{-i}\right)$. This proofs the proposition except for the statement about Pareto domination. For any $i \in t^{\prime} \backslash t, u_{i t}(e)=f(e)<u_{i t^{\prime}}\left(e^{\prime}\right)$ and for any $i \in t$, $u_{i t}(e)=u_{i}(e) \leq u_{i}\left(e_{i}, e_{-i}^{\prime}\right) \leq u_{i}\left(e_{i}^{\prime}, e_{-i}^{\prime}\right)=u_{i t^{\prime}}(e)$.

\section{References}

Aliprantis, C. D., And K. C. Border (1994): Infinite Dimensional Analysis. SpringerVerlag. 
Arrow, K. J., And F. H. Hahn (1971): General Competitive Analysis. San Francisco, HoldenDay.

Athey, S. (1996): "Comparative Statics Under Uncertainty: Single Crossing Properties and Log-Supermodularity," Discussion paper, Massachusetts Institute of Technology.

Ausubel, L. M., And R. J. Deneckere (1993): "A Generalized theorem of the maximum," Economic Theory, 3(1), 99-107.

Birkhoff, G. (1967): Lattice Theory. American Mathematical Society.

Brock, W. A. (1983): "A revised version of Samuelson's Correspondence Principle: Applications of recent results on the asymptotic stability of optimal control to the problem of comparing long run equilibria," in Models of Economic Dynamics, ed. by H. F. Sonnenschein, vol. 264 of Lecture Notes in Economics and Mathematical Systems, pp. 86-116. Springer-Verlag.

Camerer, C. F., And M. J. Knez (1994): "Creating Expectational Assests in the Laboratory: Coordination in "Weakest Link" Games," Strategic Management Journal, 15(SI), 101-119.

Cooper, R., And A. John (1988): "Coordinating Coordination Failures in Keynesian Models," Quarterly Journal of Economics, 88(103), 441-463.

DANCER, E., And P. Hess (1991): "Stability of fixed points for order preserving discrete time dynamical systems," Journal für die reine und angewandte Mathematik, 419, 125-139.

Echenique, F., and T. Sabarwal (2000): "Strong Comparative Statics of Equilibrium," Mimeo, UC Berkeley.

FARrell, J., ANd G. SAlOner (1985): "Standardization, compatibility and innovation," Rand Journal of Economics, 16(1), 70-83.

Hatta, T. (1980): "Structure of the Correspondence Principle at an Extremum Point," The Review of Economic Studies, 47(5), 987-997. 
Lippman, S. A., J. W. Mamer, and K. F. MCCardle (1987): "Comparative Statics in Non-cooperative Games via Transfinitely Iterated Play," Journal of Economic Theory, 41(2), $288-303$.

Magill, M. J., And J. A. Sheinkman (1979): "Stability of Regular Equilibria and the Correspondence Principle for symmetric variational problems," International Economic Review, $20(2), 297-315$.

Mas-Colell, A., M. D. Whinston, and J. R. Green (1995): Microeconomic Theory. Oxford University Press.

Milgrom, P., Y. Qian, and J. Roberts (1991): "Complementarities, Momentum and the Evolution of Modern Manufacturing," American Economic Review, 81(2), 84-88.

Milgrom, P., And J. Roberts (1990): "Rationalizability, Learning and Equilibrium in Games with Strategic Complementarities," Econometrica, 58(6), 1255-1277.

(1991): "Adaptive and Sophisticated Learning in Normal Form Games," Games and Economic Behavior, 3(1), 82-100.

— (1994): "Comparing Equilibria," American Economic Review, 84(3), 441-459.

Milgrom, P., And C. Shannon (1994): "Monotone Comparative Statics," Econometrica, $62(1), 157-180$.

Samuelson, P. A. (1947): Foundations of Economic Analysis. Harvard University Press.

Sobel, M. J. (1988): "Isotone Comparative Statics in Supermodular Games," mimeo, SUNY at Stony Brooks.

Topkis, D. M. (1979): "Equilibrium Points in Nonzero-Sum n-Person Submodular Games," SIAM Journal of Control and Optimization, 17(6), 773-787.

— (1998): Supermodularity and Complementarity. Princeton University Press. 
Villas-Boas, J. M. (1997): "Comparative Statics of Fixed Points," Journal of Economic Theory, 73(1), 183-198.

Vives, X. (1990): "Nash Equilibrium with Strategic Complementarities," Journal of Mathematical Economics, 19(3), 305-321.

Zhou, L. (1994): "The Set of Nash Equilibria of a Supermodular Game Is a Complete Lattice," Games and Economic Behavior, 7(2), 295-300. 